\title{
Sail Performance Analysis of Sailing Yachts by Numerical Calculations and Experiments
}

\author{
Y. Tahara, Y. Masuyama, T. Fukasawa and M. Katori \\ National Maritime Research Institute, Kanazawa Institute of Technology \\ Osaka Prefecture University, North Sails \\ Japan
}

\section{Introduction}

Sails of a sailing yacht can be considered as multiple soft thin wings (membrane wings) with relative large cambers, and are often used at large attack angles. The shape of sail is determined as an equilibrium state of both aerodynamic force and tension acting on the sail surface. In particular, a spinnaker used for the running condition is a very soft membrane like a parachute, and the shape is simply formed by self-generated aerodynamic forces which are strongly affected by the sail shape itself. These facts lead to new challenges in the present problem, i.e., in the measurements the sail shape must be accurately measured in the flying condition, and in numerical simulation of flow and forces the sail flying shape is correctly given or predicted as a part of solution. The present study concerns the authors' ongoing effort on analyses of sail performance of sailing yachts by numerical calculations and experiments, and in this paper, the focus of discussions is more on the former. Two computational fluid dynamics (CFD) methods are used in the present study, and the results are validated through detailed comparison with experimental data. The data are obtained in onboard full-scale measurements by using a sail dynamometer boat. Our study concerns both the upwind and downwind sailing conditions; however, we focus on the former in the present chapter due to the limitation of space in this book. More detailed background of the present work is well described in Masuyama et al. (2009).

One of the two CFD methods is a Vortex Lattice method (VLM). Although the VLM is a potential flow calculation, it is well known the results agree well with the measured data at the upwind condition of small attack angle. The VLM is used as the sail design and making tool due to the quick convergence ability for the parametric survey of sail shape to obtain the desired sail performance, and also due to good compatibility with the finite element method (FEM) for the strength analysis. In this paper, a method to shed wake vortices stepby-step developed by Fukasawa was adopted in the Vortex Lattice method (Fukasawa, 1993; Fukasawa \& Katori, 1993).

Another CFD method is a Multiblock Reynolds-Averaged Navier-Stokes (RANS)-based CFD named "FLOWPACK". This code was developed by Tahara specifically for CFD education and research, and design applications for ship hydrodynamics, aerodynamics, and fluid engineering (Tahara, 2008). As part of the developments for application to design problems, a complete multiblock domain decomposition feature was included. The numerical method of FLOWPACK solves the unsteady RANS and continuity equations for mean velocity and pressure. Either a zero or a two-equation turbulence model can be used for turbulence flow calculation, and in the present study the former was used. The FLOWPACK was included as a 
solver in a sail performance analyzer named "Advanced Aero Flow (AAF)" developed by Katori (Katori, 2009). The AAF is a specialized package for the calculation of sail performance of sailing yachts, and composed of both mesh generator and post analyzer.

The sail shapes and performance were measured using a sail dynamometer boat Fujin under sailing condition on the sea (Masuyama et al., 1997a, 1997b). Fujin is a 34-foot LOA boat, in which load cells and CCD cameras were installed to simultaneously measure the sail forces and shapes. At the same time, the sailing conditions of the boat, e.g., boat speed, heel angle, wind speed, and wind angle, were measured. The shapes and 3D coordinates of the sails were used for the input data of the numerical calculations, and the calculated results were compared with the measured data. The sail coordinates with aerodynamic coefficients are tabulated for some sailing conditions in order to provide benchmark data for the CFD validation.

In this paper, overview of the above-mentioned CFD methods and experiments are described. As the aforementioned, sail flying shapes are considered in the present CFD so that the accurate prediction of flow and aerodynamic forces is possible. Discussion of the results is based on detailed comparison with the measurements. The discussion also includes the current capability of the CFD methods in the present problem, and prognosis for the enhancement of the capability in future work for higher accuracy and/or more complicated flow simulation. It will be noteworthy that the overall trends of the flow and the aerodynamic forces measured in the experiments are fairly well predicted by the present computations; and at the same time, experimental techniques originally implemented and used in the present study are shown very promising and capable to provide very detailed benchmark data for CFD validation.

\section{Sail plan for the analysis}

In this study the experiments and numerical calculations were performed for the upwind sailing condition. The sail shapes and performance were measured using a sail dynamometer boat Fujin. The sail plan of the Fujin and the coordinate system are shown in Fig.1. The principal dimensions of the boat and the detailed measurements of the sails are also shown in Table 1 . The measurement system of the boat and testing conditions are described in section 5 , and the measured and calculated results are compared and discussed in section 6.
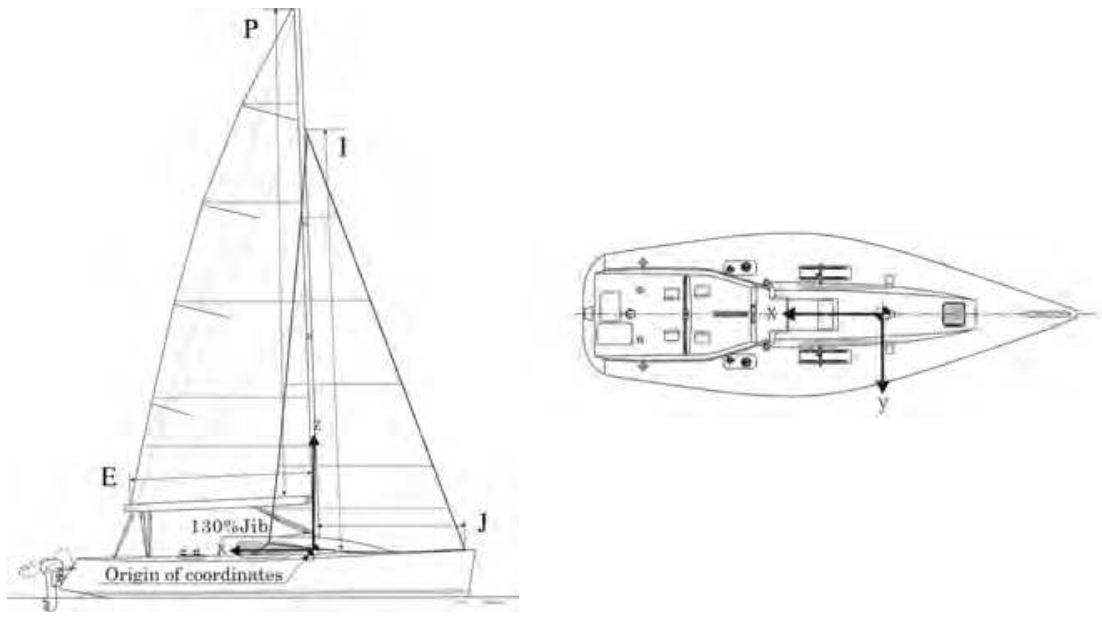

Fig. 1. Schematic showing the sail plan of Fujin with $130 \%$ jib and the coordinate system 


\begin{tabular}{|c|c|c|c|c|}
\hline \multicolumn{2}{|l|}{ HULL } & \multicolumn{3}{|c|}{ SAIL DIMENSIONS } \\
\hline Length Over All [m] & 10.35 & & Mainsail & $130 \% \mathrm{Jib}$ \\
\hline Length Water Line [m] & 8.80 & Peak Height $[\mathrm{m}]$ & 13.82 & 10.70 \\
\hline Breadth Maximum [m] & 3.37 & Luff Length [m] & 12.50 & 11.45 \\
\hline Breadth Water Line [m] & 2.64 & Foot Length [m] & 4.44 & 4.89 \\
\hline Displacement [ton] & 3.86 & Sail Area $\left[\mathrm{m}^{2}\right]$ & 33.20 & 26.10 \\
\hline \multicolumn{2}{|l|}{ SAIL } & Height [\%] & \multicolumn{2}{|c|}{ Chord Length [m] } \\
\hline $\mathrm{I}[\mathrm{m}]$ & 11.00 & 0 & 4.44 & 4.89 \\
\hline $\mathrm{J}[\mathrm{m}]$ & 3.61 & 10 & 4.13 & 4.44 \\
\hline $\mathrm{P}[\mathrm{m}]$ & 12.55 & 20 & 3.85 & 3.94 \\
\hline $\mathrm{E}[\mathrm{m}]$ & 4.51 & 40 & 3.23 & 2.94 \\
\hline \multirow{3}{*}{\multicolumn{2}{|c|}{ I, J, P, E of Sail are defined in Fig. 1.}} & 60 & 2.43 & 1.97 \\
\hline & & 80 & 1.39 & 0.98 \\
\hline & & 100 & 0.15 & 0.10 \\
\hline
\end{tabular}

Table 1. Principal dimensions of Fujin and detailed measurements of sails

\section{Overview of Vortex Lattice method (VLM)}

\subsection{Basic concept of Vortex Lattice method}

The Vortex Lattice method is a branch of CFD, and it is often used at the early stage of yacht sail design because of the comparatively less computational time. This method is based on the potential theory, similar to the panel method, and the flow around the sail is expressed by discrete vortices. The Vortex Lattice method has its root in the lifting line theory formulated by Prandtl in 1918. A wing is represented by a single vortex line in the lifting line theory, and the force acting on the wing is approximated by the force acting on the vortex line.
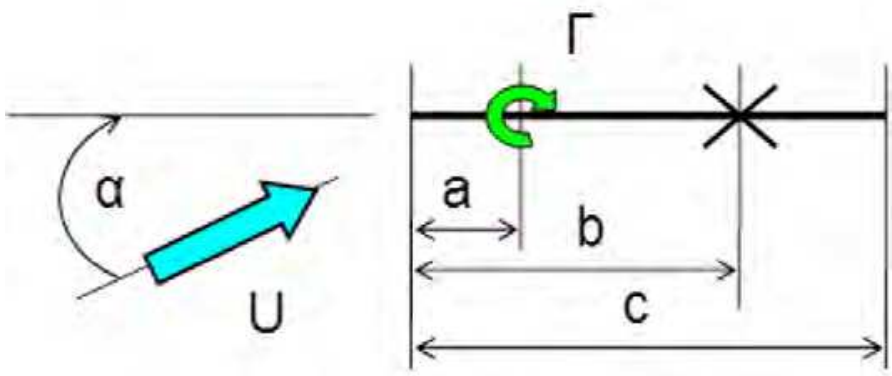

Fig. 2. 2-Dimensional flat plate wing and a vortex filament

Firstly, 2-dimensional flow around a flat plate wing is considered. A vortex filament is located at a distance " a " from the leading edge of the wing as shown in Fig.2. Although the onset flow $U$ is constant, the flow over the wing is accelerated, while it is decelerated below the wing, because of the flow induced by the vortex filament. This leads to the pressure decrease on the back surface and the pressure increase on the front surface of the wing accordingly to Bernoulli's theorem. This means that the flow around the wing can be realized by a vortex filament in the flow. The strength of vortex filament, or sometimes called circulation, is determined by a boundary condition on the wing; that is, there is no cross flow through the 
wing. This boundary condition is usually satisfied at a certain point called control point. Assuming that the control point is located at a distance " $b$ " from the leading edge of the wing shown in Fig.2, and satisfying the boundary condition at this point, the strength of the vortex filament can be determined. Once the strength of vortex filament is determined, the lift acting on the vortex filament can be calculated according to Kutta-Joukowski theorem, that is,

$$
\mathrm{L}=\rho U \Gamma
$$

where $\rho$ is the density of the fluid and $\Gamma$ is the strength of vortex filament. If the calculated lift is assumed to equal that generated in a 2-dimentional thin parabolic shape airfoil, the locations of the vortex filament and the control point are determined to be $a=c / 4$ and $\mathrm{b}=3 c / 4$, where " $\mathrm{c}$ " is the chord length of the wing. This is called 1/4-3/4 rule, which was shown by Pistolesi (Pistolesi, 1937). This rule is used as the basis of the present Vortex Lattice method.

\subsection{Application to sail configuration}

As the yacht sail is a 3-dimension shape body, attention should be paid to the treatment of the end of vortex line. According to the Helmholtz's theorem on vortex, the vortex line should expand from the boundary to the boundary of the flow or shuts oneself and makes vortex ring. Accordingly, in the 3-dimensional body, the vortex line should expand infinity from the edge of the body. In the lifting line theory, or the Vortex Lattice method, the vortex line is assumed to be a horseshoe type shown in Fig.3(a), and the vortex line changes its direction at the edge of the body to extend to infinity as trailing free vortices.

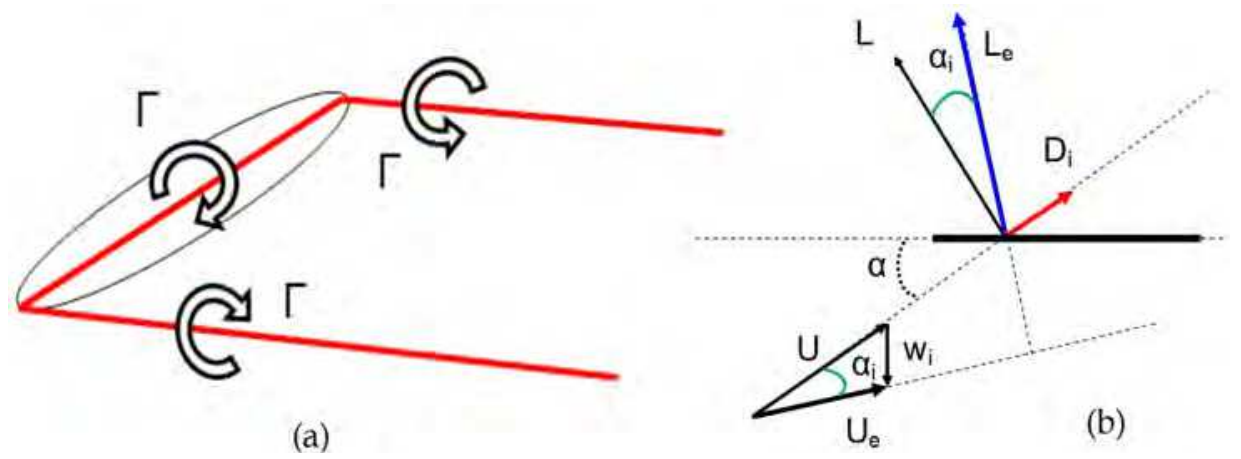

Fig. 3. Horseshoe vortex and downwash effect

In case of the horseshoe vortex shown in Fig.3(a), the flow induced by each vortex line affects the onset flow in the magnitude and the direction. According to Biot-Savart law, the velocity vector induced by a slight part of the vortex line $\mathrm{d} \ell$ is given by

$$
\overrightarrow{\mathrm{v}}=\frac{\Gamma}{4 \Pi} \int \frac{\mathrm{d} \vec{\ell} \times \overrightarrow{\mathrm{r}}}{|\overrightarrow{\mathrm{r}}|^{3}}
$$

where $\vec{r}$ is a position vector from the vortex part to the point concerned. The downward velocity called downwash $\mathrm{w}_{\mathrm{i}}$ is calculated by using Equation (2), which affects the onset flow. This causes the reduction of the attack angle of total inflow by $a_{i}$ as shown in Fig.3(b), 
and the total velocity of inflow into the wing changes to $U_{e}$. If the lift acting on the wing is defined as the force perpendicular to the onset flow direction, it is given by the following formula according to Kutta-Joukowski theorem.

$$
\mathrm{L}=\mathrm{L}_{\mathrm{e}} \cos \mathrm{a}_{\mathrm{i}} \approx \mathrm{L}_{\mathrm{e}}=\rho \mathrm{U}_{\mathrm{e}} \Gamma \approx \rho \mathrm{U} \Gamma
$$

In this case, the force in the onset flow direction is generated, which is the apparent drag called induced drag given by

$$
\mathrm{D}_{\mathrm{i}}=\mathrm{L}_{\mathrm{e}} \sin \mathrm{a}_{\mathrm{i}} \approx \mathrm{L}_{\mathrm{e}} \mathrm{a}_{\mathrm{i}}=\frac{\mathrm{L}_{\mathrm{e}}}{\mathrm{U}} \mathrm{w}_{\mathrm{i}} \approx \frac{\mathrm{L}}{\mathrm{U}} \mathrm{w}_{i}
$$

The induced drag is a distinctive drag in a 3-dimensional wing, and does not appear in a 2dimensional wing.

In the Vortex Lattice method, the lift, induced drag, and center of pressure are calculated by arranging horseshoe vortices of different strength on the surface of sail. By placing a number of horseshoe vortices, the sail of complex shape with twist, camber, or two or more sails, can be analyzed. Falkner used the name "Vortex Lattice" firstly in his report, in which a wing was covered with a grid of straight horseshoe vortices (Falkner, 1943, 1946). In 1950's, only the analysis where the trailing vortices are placed in the straight line was able to be carried out because of the computer capability, and the accuracy was questionable. It was 1965 when the Vortex Lattice method started to demonstrates its ability along with the development of computer, and the method came to be used for the performance prediction of yacht sail. The yacht sail is one of the most suitable objects for applying the Vortex Lattice method because of its thickness, if the viscous effect of fluid can be disregarded. An application of the Vortex Lattice method to the performance prediction of yacht sail will be explained in the following paragraph with the use of a step-by-step procedure to estimate the trailing vortex deformations.

Discretized horseshoe vortices are located on the sail plane in the Vortex Lattice method. It is usual to divide the sail plane into quadrilateral panels as shown in Fig.4, and the

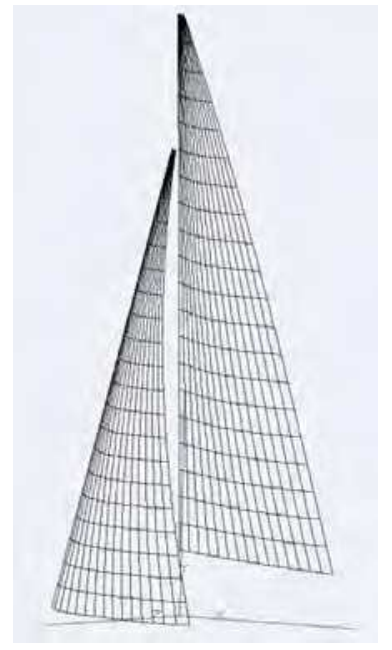

Fig. 4. Panel discretization of sails 
horseshoe vortices are placed at 1/4 length of panel from the front end edge of the panel so as to trail the trailing vortices rearwards. The strengths of the horseshoe vortices are determined by satisfying the boundary condition on the sail; that is, the total flow of the onset flow and the induced wake by vortices is parallel to the sail surface at control points. The control point is taken to be the point $3 / 4$ of length of panel from the front end edge of the panel according to the $1 / 4-3 / 4$ rule.

According to Biot-Savart law, the velocity vector at the control point of $i$-th panel induced by other vortices are given by

$$
\overrightarrow{\mathrm{v}}_{\mathrm{i}}=\frac{1}{4 \Pi} \int \frac{\Gamma(\mathrm{d} \vec{\ell} \times \overrightarrow{\mathrm{r}})}{|\overrightarrow{\mathrm{r}}|^{3}}=\sum_{\mathrm{j}} \frac{\Gamma_{\mathrm{j}}}{4 \Pi} \sum_{\mathrm{k}=1}^{3} \frac{\cos \mathrm{a}_{\mathrm{kji}}+\cos \beta_{\mathrm{kji}}}{\mathrm{h}_{\mathrm{kji}}} \overrightarrow{\mathrm{e}}_{\mathrm{kji}}
$$

where $a_{\mathrm{kji}} \beta_{\mathrm{kji}}, \mathrm{h}_{\mathrm{kji}}$, are the angles and the distance of a k-th filament of a j-th horseshoe vortex and i-th control point shown in Fig.5, which shows a plane containing the vortex filament and the control point. $\overrightarrow{\mathrm{e}}_{\mathrm{kji}}$ is a unit vector perpendicular to the plane shown in Fig.5. k=1, 2, 3 in Equation (5) denotes each vortex filament of a horseshoe vortex. With the use of Equation (5), the boundary condition on the control point can be given by

$$
\sum_{i}^{N B} \vec{v}_{i} \cdot \vec{n}=\vec{U} \cdot \vec{n}_{i}-\sum_{i}^{N W} \vec{v}_{i} \cdot \vec{n}
$$

where $\vec{U}$ is an onset flow velocity vector and $\vec{n}$ is the unit normal vector at the control points. NB is the number of bound horseshoe vortices on the sail plane and NW is the number of trailing horseshoe vortices in the wake. Equation (6) can be written in the vector matrix form by

$$
[\Lambda]\{\Gamma\}=\left\{\mathrm{u}_{\mathrm{n}}\right\}+\left\{\mathrm{v}_{\mathrm{n}}\right\}
$$

Solving Equation (7), the strength of bound vortices can be obtained.

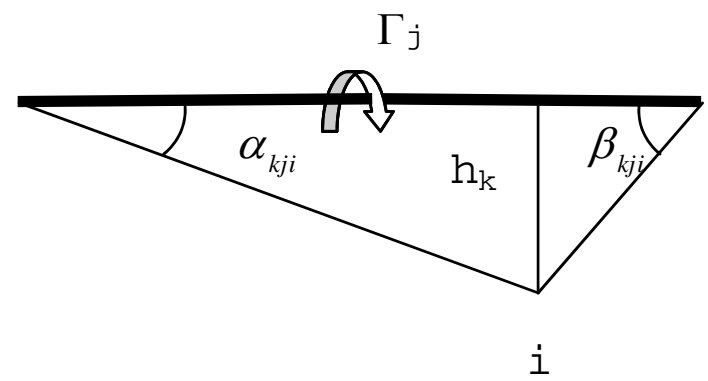

Fig. 5. K-th filament of $\mathrm{j}$-th horseshoe vortex and i-th control point

\subsection{Step-by-step vortex shedding technique}

The important point in the Vortex Lattice approach to yacht sail is the handling of the wake of the sail. The wake vortices proceed downstream from the trailing edge, or leech/foot of sail, in the Vortex Lattice method. The location of wake vortices are determined by the condition that they are free vortices; that is, the stream line of wake vortices should be 
parallel to the velocity field induced by total vortex system. A step-by-step procedure developed by Fukasawa was adopted in this paper to determine the strength of the bound vortices and the location of wake vortices (Fukasawa, 1993; Fukasawa \& Katori, 1993; Masuyama et al., 1997a, 1997b). The wake vortices are shed from the trailing edge in each time step according to Helmholtz's theorem; that is,

$$
\begin{gathered}
\Gamma_{\mathrm{B}}+\Gamma_{\mathrm{W}}=0 \\
\frac{\mathrm{D} \Gamma_{\mathrm{B}}}{\mathrm{Dt}}=0
\end{gathered}
$$

where $\Gamma_{\mathrm{B}}$ and $\Gamma_{\mathrm{W}}$ are the total strength of bound vortices, or the circulation around the sail, and the strength of wake vortices, respectively. From Equation (9), we have

$$
\frac{\partial \Gamma_{\mathrm{B}}}{\partial \mathrm{t}}=\tilde{\mathrm{U}} \frac{\partial \Gamma_{\mathrm{B}}}{\partial \xi}
$$

and substituting Equation (10) into (8), we have

$$
\frac{\partial \Gamma_{\mathrm{B}}}{\partial \mathrm{t}}=-\tilde{\mathrm{U}} \frac{\partial \Gamma_{\mathrm{W}}}{\partial \xi}
$$

where $\xi$ is taken to the downstream direction, and $\tilde{U}$ is the local velocity at the wave vortex. Assuming that the wake vortices proceeds $\Delta \xi$ downstream in a time step $\Delta \mathrm{t}$ with the velocity $\tilde{U}$, the strength of wake vortex shed at time step $k$, can be given by integrating Equation (11), that is,

$$
\Delta \Gamma_{\mathrm{W}}^{\mathrm{k}}=-\frac{1}{\tilde{\mathrm{U}}} \int_{0}^{\Delta \mathrm{t}} \frac{\partial \Gamma_{\mathrm{B}}}{\partial \mathrm{t}} \mathrm{d} \xi=-\frac{\Delta \xi}{\tilde{\mathrm{U}} \Delta \mathrm{t}}\left(\Gamma_{\mathrm{B}}^{\mathrm{k}}-\Gamma_{\mathrm{B}}^{\mathrm{k}-1}\right)=\Gamma_{\mathrm{B}}^{\mathrm{k}-1}-\Gamma_{\mathrm{B}}^{\mathrm{k}}
$$

Equation (12) means that the strength of wake vortex shed at time step $\mathrm{k}$ is the increase of the strength of bound vortex from time step k-1 to time step k. Once a vortex filament is shed at time step $\mathrm{k}$, it proceeds downstream with a constant strength according to the local field velocity, i.e., each horseshoe wake vortex moves in the direction of field velocity in each time step. The field velocity is updated in every time step. The calculation is carried forward until the the calculated lift and drag forces converges. The forces vector and the moment acting on the sail are calculated accordingly to Kutta-Joukowski theorem.

$$
\overrightarrow{\mathrm{F}}=\rho \int \Gamma \tilde{U} \times \mathrm{d} \overrightarrow{\mathrm{s}}
$$

with the use of the vortex strengths of the wake vortices and the bound vortices determined by solving Equation (7).

Finally, the overall numerical solution procedure of the present Vortex Lattice method is summarized as follows:

Step 1. Divide the sail planes into quadrilateral panels, and allocate horseshoe vortices on the sail plane.

Step 2. Input the mast rake angle, heel angle of the yacht, apparent wind speed and apparent wind angle. 
Step 3. Solve the strength of bound horseshoe vortices on the sail plane with nondeformed trailing vortices.

Step 4. Compute the total circulation around the sail caused by horseshoe vortices.

Step 5. Compute the increment of the total circulation and shed free horseshoe vortices according to Equation (12).

Step 6. Calculate the local velocities along the wake, and deform the trailing vortices.

Step 7. Compute the force vector and moment acting on the sails.

Step 8. Solve the strength of bound horseshoe vortices on the sail plane with trailing free vortices.

Step 9. Repeat Step 4 through Step 8 until the force is converged.

Fig. 6 shows the example calculation results. In the present study, the mast and rigging were not considered for the series calculations, and the mirror image was taken into account about the deck plane of the boat. Since the vortex lattice methods do not predict viscous drag, the viscous drag acting on the sails and rigging was calculated empirically using a drag coefficient $C_{D p}$. The value of $C_{D p}$ was obtained from the measured data in the previous papers and formulated for the upwind condition as follows:

$$
\mathrm{C}_{\mathrm{Dp}}=0.0026 \gamma_{\mathrm{A}}+0.005
$$

where $\gamma_{A}$ is apparent wind angle in degrees.

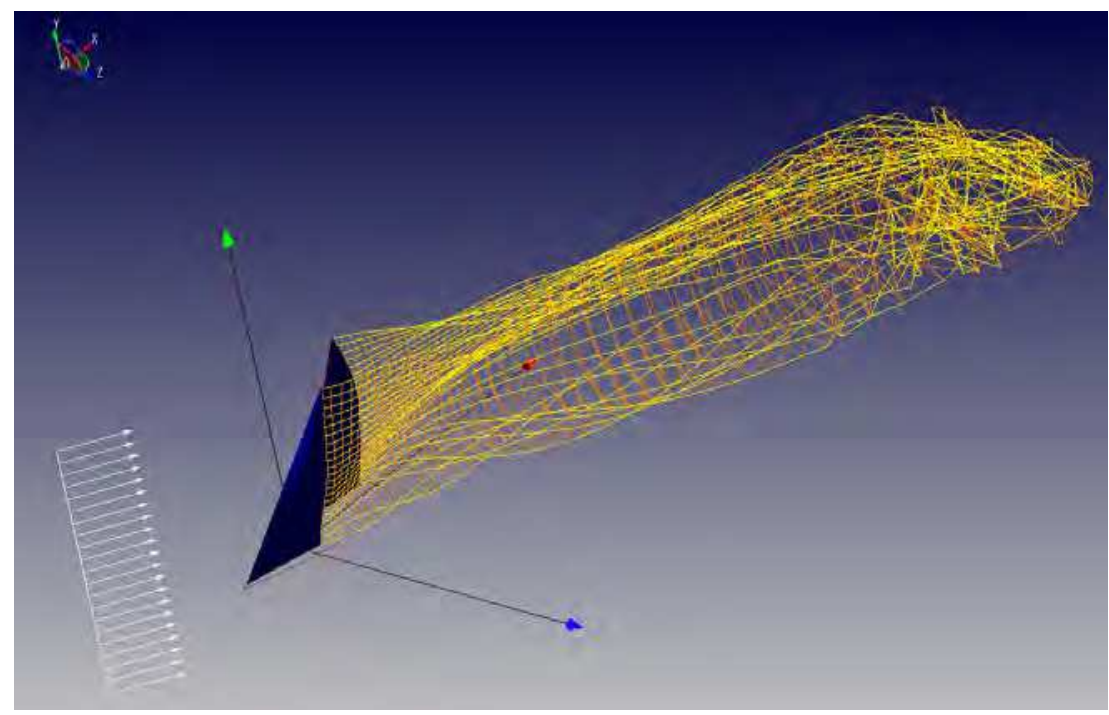

Fig. 6. Calculated wake by Vortex Lattice method

\section{Overview of Reynolds-averaged Navier-Stokes equation method}

The RANS-based CFD method used in the present study was FLOWPACK. The code was developed by Tahara specifically for CFD education and research and for design applications for ship hydrodynamics, aerodynamics, and fluid engineering. As part of the developments for application to design problems, a complete multiblock domain 
decomposition feature was included. At present, FLOWPACK has a good interface with the authors' inhouse automatic grid generator as well as with commercial grid generation software. For a complete documentation of the method is available in Tahara (2008). In the following, an overview of the numerical method is given.

\subsection{Governing equations}

Let us consider a sail system fixed in the uniform onset flow (see Fig.1 for the basic coordinate system). The non-dimensional RANS equations for unsteady, three-dimensional incompressible flow can be written in Cartesian tensor notation as

$$
\begin{gathered}
\frac{\partial U_{i}}{\partial t}+U_{j} \frac{\partial U_{i}}{\partial x^{j}}+\frac{\partial \overline{u_{i} u_{j}}}{\partial x^{j}}+\frac{\partial p}{\partial x^{i}}-\frac{1}{\operatorname{Re}} \nabla^{2} U_{i}=0 \\
\frac{\partial U_{i}}{\partial x^{i}}=0
\end{gathered}
$$

where $U_{i}(i=1,2,3)=(U, V, W)$ and $u_{i}(i=1,2,3)=(u, v, w)$ are the Cartesian components of mean and fluctuating velocities, respectively, normalized by the reference velocity $U_{0}, x^{i}(i=1,2,3)$ $=(X, Y, Z)$ is the dimensionless coordinates normalized by a characteristic length $L, \operatorname{Re}=U_{0} L / v$ is the Reynolds number, $v$ is the kinematic viscosity, the barred quantities $-\overline{u_{i} u_{j}}$ are the Reynolds stresses normalized by $U_{0}^{2}$, and $p$ is the pressure normalized by $\rho U_{0}^{2}$. If $-\overline{u_{i} u_{j}}$ are related to the corresponding mean rate of strain through an isotropic eddy viscosity $v_{t}$, i.e.,

$$
-\overline{u_{i} u_{j}}=v_{t}\left(\frac{\partial U_{i}}{\partial x^{j}}+\frac{\partial U_{j}}{\partial x^{i}}\right)-\frac{2}{3} \delta_{i j} k
$$

where $k=(\overline{u u}+\overline{v v}+\overline{w w}) / 2$ is the turbulent kinetic energy, Equation (15) becomes

$$
\frac{\partial U_{i}}{\partial t}+\left(U_{j}-\frac{\partial v_{t}}{\partial x^{j}}\right) \frac{\partial U_{i}}{\partial x^{j}}-\frac{\partial v_{t}}{\partial x^{j}} \frac{\partial U_{j}}{\partial x^{i}}+\frac{\partial p}{\partial x^{i}}\left(p+\frac{2}{3} k\right)-\frac{1}{R_{\phi}} \nabla^{2} U_{i}=0
$$

where $1 / R_{\phi}=1 / \operatorname{Re}+v_{t}$, and $\phi=U_{i}(i=1,2,3)$. Equations (16) and (18) can be solved for $U_{i}$ and $p$ when a suitable turbulence model is employed to calculate the eddy-viscosity distribution. Either a zero or a two-equation turbulence model can be used for turbulent flow calculation, and a model used for the present study is the former, i.e., Baldwin-Lomax model (Baldwin \& Lomax, 1978), which is an algebraic scheme that makes use of a two-layer isotropic eddyviscosity formulation. Detailed validation study of this model for boundary layer flows around three-dimensional bodies was done by the author (Tahara, 1995; Tahara \& Stern, 1996). In this model, the eddy viscosity is evaluated as follows:

$$
\begin{cases}\left(v_{t}\right)_{\text {inner }} & y \leq y_{c} \\ \left(v_{t}\right)_{\text {outer }} & y>y_{c}\end{cases}
$$

where $y$ is the distance normal to the wall surface and $y_{c}$ is the minimum value of $y$ where both the inner and outer viscosities match. The inner viscosity follows the Prandtl-Van 
Driest formula, i.e., $\left(v_{t}\right)_{\text {inner }}=\lambda^{2}|\omega|$, where $\lambda=\kappa y\left[1-\exp \left(-y^{+} / A^{+}\right)\right]$is the turbulent length scale for the inner region, $\kappa$ and $A^{+}$are model constants, $|\omega|$ is the vorticity magnitude, and $y^{+}$is the dimensionless distance to the wall. In the outer region, eddy viscosity is given by $\left(v_{\mathrm{t}}\right)_{\text {outer }}=K C_{\mathrm{cp}} F_{\text {wake }} F_{\text {Kleb }}$, where $K$ and $C_{\mathrm{cp}}$ are model constants, $F_{\text {wake }}=\min \left(y_{\max } F_{\text {max }}\right.$ $\left.C_{\mathrm{wk}} y_{\max } U_{\mathrm{dif}^{2}}{ }^{2} F_{\max }\right)$, and $F_{\mathrm{Kleb}}=\left[1+5.5\left(C_{\mathrm{Kleb}} y / y_{\max }\right)^{6}\right]^{-1}$. The $F_{\max }$ and $y_{\max }$ are determined by the value and corresponding location, respectively, of the maximum of $F=y|\omega|[1-\exp (-$ $\left.\left.y^{+} / A^{+}\right)\right]$. The quantity $U_{\text {dif }}$ is the difference between maximum and minimum velocity magnitudes in the profile and is expressed as $U_{\text {dif }}=\left(U^{2}+V^{2}+W^{2}\right)_{\max }^{1 / 2}-\left(U^{2}+V^{2}+W^{2}\right)_{\min }^{1 / 2}$. $C_{\text {Kleb }}$ and $C_{\mathrm{wk}}$ are additional model constants. Numerical values for the model constants are $A^{+}=26, \kappa=0.4, K=0.0168, C_{\mathrm{cp}}=1.6, C_{\mathrm{wk}}=1.0$, and $C_{\mathrm{Kleb}}=0.3$.

\subsection{Discretization and velocity-pressure coupling}

In the following, discretization and velocity-pressure coupling of the present RANS method are described. First, it is convenient to rewrite the transport equations for momentum $\left(U_{i}\right)$ in the following general form:

$$
\nabla^{2} \phi=R_{\phi}\left[\sum_{j=1}^{3}\left(U_{j}-\frac{1}{\sigma_{\phi}} \frac{\partial v_{t}}{\partial x^{j}}\right) \frac{\partial \phi}{\partial x^{j}}+\frac{\partial \phi}{\partial t}\right]+s_{\phi}
$$

where $\phi$ again represents any one of the convective transport quantities $\left(U_{i}\right)$, and $s_{\phi}$ is the source function for the corresponding quantity. We transform the physical space $\left(x^{i}, t\right)$ into a rectangular region in the computational space $\left(\xi^{i}, \tau\right)$ using the following coordinate transformations:

$$
\begin{array}{cc}
t=\tau, \quad x^{i}=x^{i}\left(\xi^{j}\right), & e_{i} \cdot \nabla \phi=\frac{1}{J} \sum_{j=1}^{3} b_{i}{ }^{j} \frac{\partial \phi}{\partial \xi^{j}} \\
\nabla^{2} \phi=\sum_{i=1}^{3} \sum_{j=1}^{3} g^{i j} \frac{\partial^{2} \phi}{\partial \xi^{i} \xi^{j}}+\sum_{j=1}^{3} f^{j} \frac{\partial \phi}{\partial \xi^{j}}, & \frac{\partial \phi}{\partial t}=\frac{\partial \phi}{\partial \tau}-\frac{1}{J} \sum_{i=1}^{3} \sum_{j=1}^{3} b_{i}{ }^{j} \frac{\partial x^{i}}{\partial \tau} \frac{\partial \phi}{\partial \xi^{j}}
\end{array}
$$

Then the continuity equation (16) and the transport equations (20) for momentum parameters can be written as

$$
\begin{gathered}
\frac{1}{J} \sum_{i=1}^{3} \sum_{j=1}^{3} \frac{\partial}{\partial \xi^{i}}\left(b_{j}^{i} U_{j}\right)=0 \\
\sum_{j=1}^{3}\left(g^{j j} \frac{\partial^{2} \phi}{\partial \xi^{j} \partial \xi^{j}}-2 a_{\phi}^{j} \frac{\partial \phi}{\partial \xi^{j}}\right)=R_{\phi} \frac{\partial \phi}{\partial \tau}+S_{\phi}
\end{gathered}
$$

where

$$
2 a_{\phi}^{j}=\frac{R_{\phi}}{J} \sum_{n=1}^{3} b_{n}^{j}\left(U_{n}-\frac{\partial x^{n}}{\partial \tau}-\frac{1}{J \sigma_{\phi}} \sum_{m=1}^{3} b_{n}^{m} \frac{\partial v_{t}}{\partial \xi^{m}}\right)-f^{j}
$$




$$
S_{\phi}=s_{\phi}-2\left(g^{12} \frac{\partial^{2} \phi}{\partial \xi^{1} \partial \xi^{2}}+g^{13} \frac{\partial^{2} \phi}{\partial \xi^{1} \partial \xi^{3}}+g^{23} \frac{\partial^{2} \phi}{\partial \xi^{2} \partial \xi^{3}}\right)
$$

The geometric coefficients $b_{i}^{j}, g^{i j}$, and $f^{j}$ appearing in the above equations are defined by Thompson et al. (1985). The transport equations (22) on a computational cell (shown in Fig.7(a)) are linearized and evaluating coefficients and source term at the center node $\mathrm{P}$ of the element yields

$$
\sum_{j=1}^{3}\left(g_{\mathrm{P}}^{j j} \frac{\partial^{2} \phi}{\partial \xi^{j} \partial \xi^{j}}-2\left(a_{\phi}^{j}\right)_{\mathrm{P}} \frac{\partial \phi}{\partial \xi^{j}}\right)=\left(R_{\phi}\right)_{\mathrm{P}} \frac{\partial \phi}{\partial \tau}+\left(S_{\phi}\right)_{\mathrm{P}}
$$

or

$$
g_{\mathrm{P}}^{11} \phi_{\xi^{1} \xi^{1}}+g_{\mathrm{P}}^{22} \phi_{\xi^{2} \xi^{2}}+g_{\mathrm{P}}^{33} \phi_{\xi^{3} \xi^{3}}=2\left(C_{\phi}\right)_{\mathrm{P}} \phi_{\xi^{1}}+2\left(B_{\phi}\right)_{\mathrm{P}} \phi_{\xi^{2}}+2\left(A_{\phi}\right)_{\mathrm{P}} \phi_{\xi^{3}}+\left(R_{\phi}\right)_{\mathrm{P}} \phi_{t}+\left(S_{\phi}\right)_{\mathrm{P}}
$$

(a)

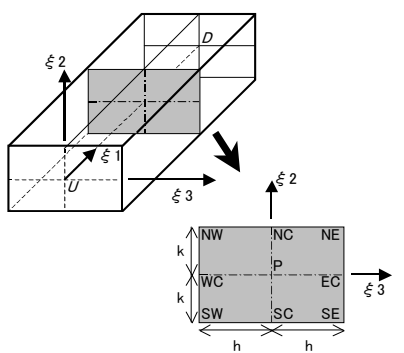

(b)

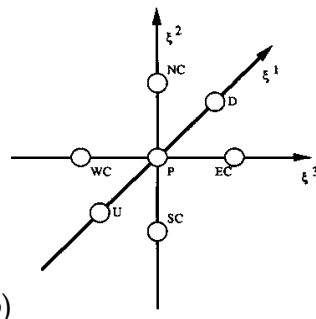

(c)

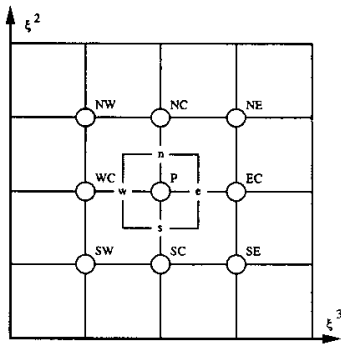

Fig. 7. Definition sketch of a computational cell (a), and nodes in regular grid (b) and continuity cell (c).

The dimensions of the computational cell are $2 l \times 2 k \times 2 h$, where $l=1 / \sqrt{g_{P}^{11}}, k=1 / \sqrt{g_{P}^{22}}$, and $h=1 / \sqrt{g_{P}^{33}}$. The above equation is discretized by the finite-analytic scheme. Solution dependent coefficients are analytically derived by solving the above linearized transport equation using a hybrid method which combines a two-dimensional analytic solution in $\xi^{2} \xi^{3}$ plane with one dimensional analytic solution in the $\xi^{1}$ direction. By specifying boundary conditions on the faces of the cell as a combination of exponential and linear functions, which are the natural solutions for the linearized transport equation, Equation (24) can be solved by the method of separation of variables. When the solution is evaluated at the center node $\mathrm{P}$ of the element, the following twelve-point finite analytic formula is obtained:

$$
\phi_{P}^{n}=\frac{1}{1+C_{\mathrm{P}}\left(C_{\mathrm{U}}+C_{\mathrm{D}}+\frac{R \phi}{\Delta \tau}\right)}\left[\begin{array}{l}
C_{\mathrm{NE}} \phi_{N E}^{n}+C_{\mathrm{NW}} \phi_{N W}^{n}+C_{\mathrm{SE}} \phi_{S E}^{n}+C_{\mathrm{SW}} \phi_{S W}^{n} \\
+C_{\mathrm{EC}} \phi_{E C}^{n}+C_{\mathrm{WC}} \phi_{W C}^{n}+C_{\mathrm{NC}} \phi_{N C}^{n}+C_{\mathrm{SC}} \phi_{S C}^{n} \\
+C_{\mathrm{P}}\left(C_{\mathrm{U}} \phi_{U}^{n}+C_{\mathrm{D}} \phi_{\mathrm{D}}^{n}+\frac{R_{\phi}}{\Delta \tau} \phi_{\mathrm{P}}^{n-1}\right)-C_{\mathrm{P}}\left(S_{\phi}\right)_{\mathrm{P}}
\end{array}\right]
$$

where 


$$
\begin{gathered}
C_{\mathrm{U}}=\frac{C e^{C l}}{l \sinh C l}, C_{\mathrm{D}}=\frac{C e^{-C l}}{l \sinh C l}, C_{\mathrm{SC}}=\frac{e^{B k}}{2 \cosh B k} P_{A}, C_{\mathrm{NC}}=e^{-2 B k} C_{\mathrm{SC}} \\
C_{\mathrm{WC}}=\frac{e^{A h}}{2 \cosh A h} P_{B}, C_{\mathrm{EC}}=e^{-2 A h} C_{\mathrm{WC}}, C_{\mathrm{SW}}=\frac{e^{A h+B k}}{4 \cosh A h \cosh B k}\left(1-P_{A}-P_{B}\right) \\
C_{\mathrm{SE}}=e^{-2 A h} C_{\mathrm{SW}}, C_{\mathrm{NW}}=e^{-2 B k} C_{\mathrm{SW}}, C_{\mathrm{NE}}=e^{-2 A h-2 B k} C_{\mathrm{SW}} \\
C_{\mathrm{P}}=\frac{h \tanh A h}{2 A}\left(1-P_{A}\right)=\frac{k \tanh B k}{2 B}\left(1-P_{B}\right), P_{A}=4 E_{2} A h \cosh A h \cosh B k \operatorname{coth} A h \\
P_{B}=1+\frac{B h \operatorname{coth} B k}{A k \operatorname{coth} A h}\left(P_{A}-1\right), E_{2}=\sum_{m=1}^{\infty} \frac{-(-1)^{m}\left(\lambda_{m} h\right)}{\left[(A h)^{2}+\left(\lambda_{m} h\right)^{2}\right]^{2} \cos \sqrt{A^{2}+B^{2}+\lambda_{m}{ }^{2}} k} \\
\lambda_{m} h=\left(m-\frac{1}{2}\right) \pi
\end{gathered}
$$

The subscripts $\mathrm{P}, \mathrm{U}$ and $\mathrm{D}$ denote the center, upstream and downstream nodes, respectively, and NC, NW, WC, etc. denote the nodes in the $\xi^{2} \xi^{3}$-plane in terms of compass directions. The superscripts (n) and (n-1) refer to the current and previous time levels, and $\Delta \tau$ is the time step. The solution of the complete flow equations involves a global iteration process, in which the velocity-pressure coupling is effected by PISO-type predictor-corrector steps. The pressure equation is derived by introducing pseudo-velocities at staggered locations while maintaining the regular grid arrangement for all the transport equations. Fig.7(b) and (c) show the locations of nodes in the regular grid in the $\xi^{2} \xi^{3}$-plane. All transport quantities and pressure are evaluated at the regular nodes. In deriving the pressure equation, a control volume is employed as a continuity cell, to establish the coupling between the velocity and pressure fields. The pressure equation used in this study is written as

$$
\begin{aligned}
\left(E_{\mathrm{d}}^{11}\right. & \left.+E_{\mathrm{u}}^{11}+E_{\mathrm{n}}^{22}+E_{\mathrm{s}}^{22}+E_{\mathrm{e}}^{33}+E_{\mathrm{w}}^{33}\right) p_{\mathrm{P}} \\
& =E_{\mathrm{d}}^{11} p_{\mathrm{D}}+E_{\mathrm{u}}^{11} p_{\mathrm{U}}+E_{\mathrm{n}}^{22} p_{\mathrm{NC}}+E_{\mathrm{s}}^{22} p_{\mathrm{SC}}+E_{\mathrm{e}}^{33} p_{\mathrm{EC}}+E_{\mathrm{w}}^{33} p_{\mathrm{WC}}-\hat{D}
\end{aligned}
$$

with

$$
\hat{D}=\hat{U}_{\mathrm{d}}^{1}-\hat{U}_{\mathrm{u}}^{1}+\hat{U}_{\mathrm{n}}^{2}-\hat{U}_{\mathrm{s}}^{2}+\hat{U}_{\mathrm{e}}^{3}-\hat{U}_{\mathrm{w}}^{3}
$$

Here $E^{i j}$ and a modified pseudovelocity $\hat{U}^{i}$ at the regular node are

$$
E^{i j}=\frac{R C_{\mathrm{P}}}{J\left[1+C_{\mathrm{P}}\left(C_{\mathrm{U}}+C_{\mathrm{D}}+\frac{R}{\Delta \tau}\right)\right]} \sum_{m=1}^{3} b_{m}^{i} b_{m}^{j}, \hat{U}^{i}=\sum_{n=1}^{3} b_{n}^{i} \hat{U}_{n}-E^{i j} \frac{\partial p}{\partial \xi^{j}}-E^{i k} \frac{\partial p}{\partial \xi^{k}}
$$

where $\hat{U}_{i}$ is a pseudovelocity given by the decomposition of Equation (25) for $U_{i}$ into $\hat{U}_{i}$ plus the pressure gradient terms, such that 


$$
U_{i}=\hat{U}_{i}-\frac{R C_{\mathrm{P}}}{J\left[1+C_{\mathrm{P}}\left(C_{\mathrm{U}}+C_{\mathrm{D}}+\frac{R}{\Delta \tau}\right)\right]} \sum_{j=1}^{3} b_{i}^{j} \frac{\partial p}{\partial \xi^{j}}
$$

The coefficients and the modified pseudovelocities in the above equations are defined at the staggered node, and obtained from those at the regular node by the one-dimensional linear interpolation. The solution of the complete flow equations involves a global iteration process, in which the velocity-pressure coupling is effected by predictor-corrector steps. In the predictor step, the pressure field at the previous time step is used in the solution of the implicit equations (25) to obtain the corresponding velocity field. Since the velocity field generally does not satisfy mass conservation, a corrector step is needed. In the corrector step, the explicit momentum equations (28) and the implicit pressure equation (26) are solved iteratively to ensure the satisfaction of the continuity equation.

\subsection{Multiblock (domain decomposition) capability, and overall numerical solution procedure}

As mentioned earlier, the multiblock (domain decomposition) capability is facilitated in the present RANS method. This capability is essential for simulation of flow around complex geometry, e.g., multiple sail system for sailing yacht as focused in the present study. Fig.8 shows overview of the present multiblock computational grid, while the grid is generated by using an automatic gridding scheme developed by the present author (Masuyama et al., 2009) Note that the gridding engine together with the present RANS method was recently implemented into a comprehensive sail performance prediction software "Advanced Aero Flow" (Katori, 2009). See the reference for more details of the scheme. Total number of grids is around a half million, and the number of multiblock is 48 . Free-stream, symmetry, and wall-surface (no slip) boundary conditions are imposed on outer and top boundaries, bottom boundary, and sail surface boundary, respectively. For the results shown in this paper, the mast and rigging are not considered in the series calculations, and the bottom boundary is located at the same height as that of deck plane of the boat (see Masuyama et al., 2009, for the results for which mast influences in computation are considered).
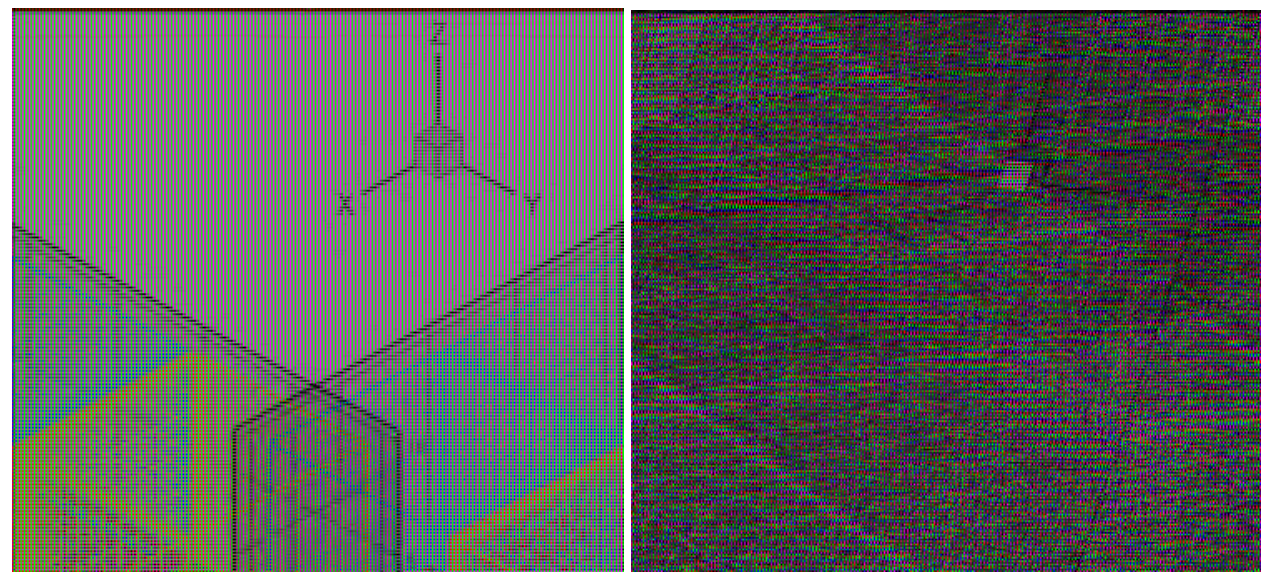

Fig. 8. Overview of the present multiblock computational grid. 
The basic strategy to handle the multiblock follows domain decomposition technique to solve the elliptic PDE by using several subdomains. After adequate discretization is applied and a simple preconditioner is introduced, the discrete alternating Schwarz's method to solve the PDE is used for boundary matching. Finally, Fig.9 shows the code structure of the present RANS method, and the overall numerical solution procedure of the present RANS method is summarized as follows:

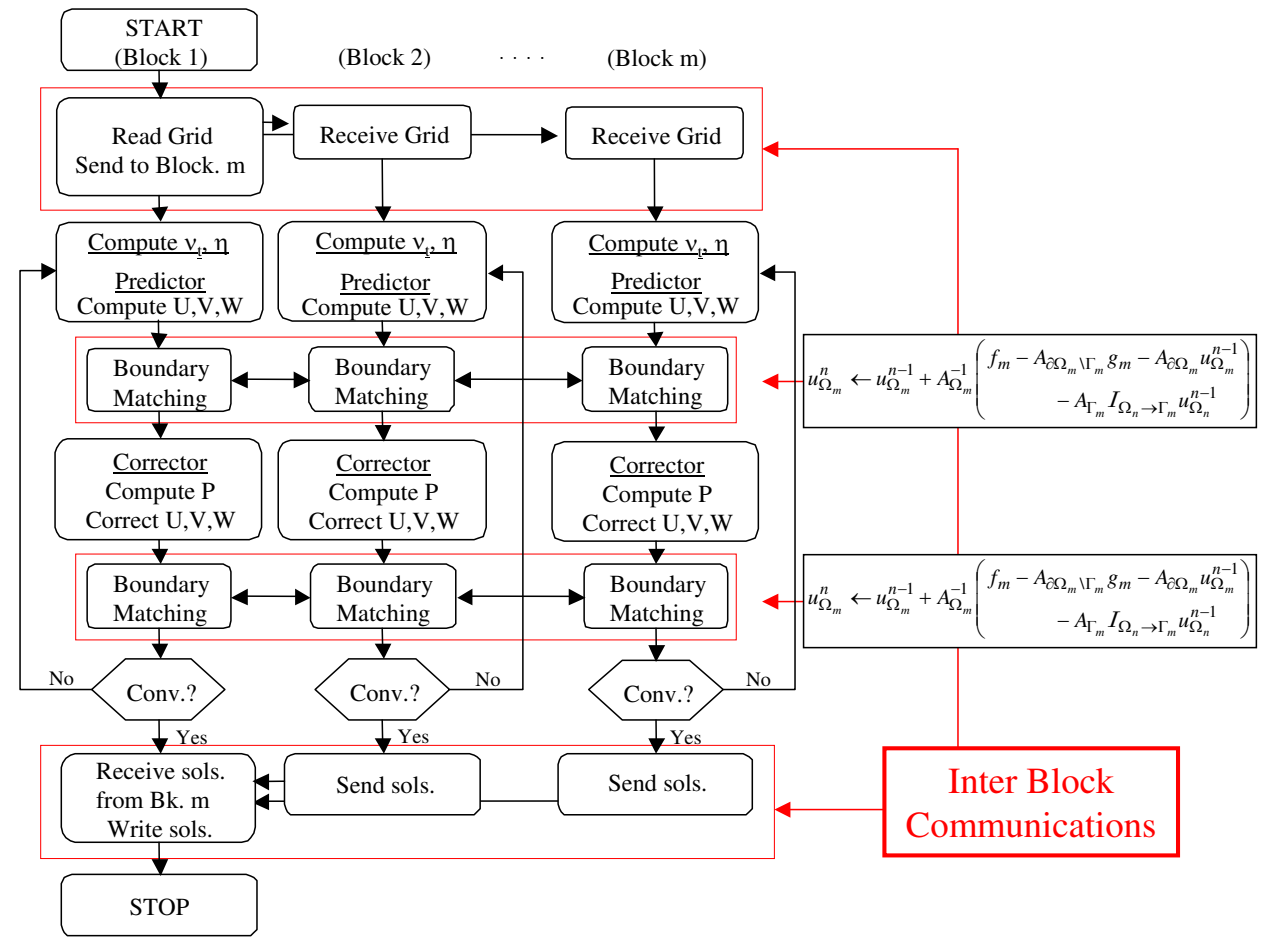

Fig. 9. PISO type solution algorithm for the present multiblock RANS method.

Step 1. Input the computational grid, setup parameters, and boundary condition information.

Step 2. Specify the initial conditions for the velocity, pressure and turbulence fields.

Step 3. Compute the geometric coefficients.

Step 4. Compute the finite-analytic coefficients for the transport equation.

Step 5. Compute eddy viscosity distribution.

Step 6. Solve transport equation for velocities $(U, V, W)$ using the previous pressure field (predictor stage for the velocity field).

Step 7. Compute the coefficients of pressure equation.

Step 8. Solve pressure equation.

Step 9. Using the newly obtained pressure, calculate the new velocity field explicitly (corrector stage for the velocity field).

Step 10. Update the finite-analytic coefficients for the transport equation for velocities $(U, V, W)$. 
Step 11. Repeat Step 8 through Step 10 for the specified number of times.

Step 12. Return to Step 4 for the next time step, until the time step reaches the given maximum value.

More details of the present RANS method are described in Tahara et al. (2006a, 2006b) in addition to the above-cited references.

\section{Measurements of upwind sail performance in full-scale condition using sail dynamometer boat Fujin}

\subsection{Full-scale measurements}

Full-scale onboard measurements are free from scale-effect problem by wind tunnel tests and appear more promising, but the challenge becomes how to accurately measure forces acting on the sail. Such studies on sail force measurements were performed by Milgram et al. (1993), Masuyama et al. (1997a, 1997b), and Hochkirch et al. (1999), who built full-scale boats with onboard sail dynamometer systems.

Milgram (1993) showed in his pioneering work that the sail dynamometer boat, Amphetrete, is quite capable. This measurement system consists of a 35-foot boat with an internal frame connected to the hull by six load cells, which were configured to measure all forces and moments acting on the sails. In his work, the sail shapes were also measured and used for CFD analyses; however unfortunately, details of the sail shape and performance data were not presented. Hochkirch et al. (1999) also built a 33-foot dynamometer boat DYNA. The aerodynamic forces acting on the sail were measured and compared with the results from wind tunnel tests (Hansen et al. 2003). The measured data were also used as input to the CFD calculation and a parametric survey was carried out (Krebber et al. 2006). Masuyama and Fukasawa were encouraged by Milgram's work, and built a sail dynamometer boat, Fujin. The measurement system installed in the Fujin and the results of calibration test and sailing test were reported by Masuyama et al. (1997a and 1997b).

\subsection{Measurements by sail dynamometer boat Fujin}

The Fujin was originally built for conducting tests on sails for the Japanese America's Cup entry in 1994. Fujin is a $10.3 \mathrm{~m}$-long ocean cruiser with a sail dynamometer system in the hull which can directly measure sail forces and moments. Fig. 1 shows the general arrangement of the Fujin. The test sails were made to correspond to a typical sail plan for an International Measurement System (IMS) class boat. The rigging of the Fujin was originally designed for testing sails for the International America's Cup Class (IACC) boat. The jib of IACC boat is relatively small. Therefore, the longitudinal position of the jib rail track of the Fujin was located further forward than that of the typical IMS boat. For this reason, the tests were performed using a fully batten mainsail and a $130 \%$ jib instead of a $150 \%$ jib. The sails were made by North Sails Japan. The axes system is also shown in Fig. 1. The origin is located on the vessel's centerline at the aft face of the mast ( $x$-direction), and the height of deck level at the base of the forestay ( $z$-direction). Table 1 shows the principal dimensions of the boat and the detailed measurements of the sails, where " $\mathrm{I}$ ", " $\mathrm{J}$ ", " $\mathrm{P}$ " and " $\mathrm{E}$ " are the measurement lengths of sail dimensions for the IMS rule as defined in Fig.1.

The aerodynamic coefficients and the coordinates of the center of effort of the sails are defined as follows: 


$$
C_{X}=\frac{X_{S}}{\frac{1}{2} \rho_{a} U_{A}{ }^{2} S_{A}}, \quad C_{Y}=\frac{Y_{S}}{\frac{1}{2} \rho_{a} U_{A}{ }^{2} S_{A}}, \quad x_{C E}=\frac{N_{S}}{Y_{S}}, \quad z_{C E}=\frac{K_{S}}{Y_{S}}
$$

where $X_{S}$ and $Y_{S}$ are the force components along the $x$ and $y$ axes of the boat respectively, and $K_{S}$ and $N_{S}$ are the moments around the $x$ and $z$ axes. $x_{C E}$ and $z_{C E}$ are the $x$ and $z$ coordinates of the center of effort of the sails (CE). The thrust force coefficient $C_{X}$ is expressed as positive for the forward direction and the side force coefficient $C_{Y}$ is positive for both port and starboard directions. It should be noted that the coordinates are given in the body axes system. Therefore, when the boat heels the $Y_{S}$ force component is not in the horizontal plane but is normal to the mast. The aerodynamic forces acting on the mast and rigging are included in the measured sail forces.

\subsubsection{Measurement system of aerodynamic performance and sail shape}

The sail dynamometer system is composed of a rigid aluminum frame and four load cells. The frame is separated structurally from the hull and connected to it by the load cells. The general arrangement of the dynamometer frame is given in Fig.10. The load cells are numbered in the figure. Two of these are 1-component load cells and the others are 2component ones. Hence, these load cells form a 6-component dynamometer system, and their outputs can be transformed to the forces and moments about the boat axes using a calibration matrix. All rig components such as mast, chain plates, winches, lead blocks, etc. are attached to the aluminum frame. The under deck portion of the mast is held by the frame, and the other rig components are attached to the frame through the deck holes. The data acquisition system and calibration method for the Fujin were described by Masuyama et al. (1997a and 1997b).

The sail shape was recorded using pairs of CCD cameras. The lower part of the mainsail was photographed using the CCD camera pair designated A in Fig.11. These were located at the mast top, $50 \mathrm{~cm}$ transversely from each side of the mast. The upper part of the mainsail was photographed using a portable video camera from below the boom. The lower part of the jib was photographed using the camera pair designated B in Fig.11, which were located at the intersection point of the forestay and the mast, $10 \mathrm{~cm}$ transversely from each side of the mast. The upper part of the jib was photographed using a portable video camera from inside the bow hatch. For measuring convenience, horizontal stripes were drawn on the mainsail and jib at heights of 10,20,40, 60 and $80 \%$ of each sail. The sail shape images were analyzed using the sail shape analyzing software, SSA-2D, developed by Armonicos Co. Fig.12(a) shows an example of processed image of the mainsail using the SSA-2D. This software calculates the curvature of the sail section by marking several points of the sail stripe and the reference line on the PC display, and indicates the parameters such as chord length, maximum draft, maximum draft position, entry angle at the luff, i.e., leading edge, and exit angle at the leech, i.e., trailing edge, as shown in Fig.12(b). The apparent wind speed (AWS) and apparent wind angle (AWA) are measured by an anemometer attached on the "Bow unit" as shown in Fig.11. This unit post can rotate freely to maintain its vertical attitude when the boat heels in order to measure the wind data in the horizontal plane. The height of the anemometer coincides with the geometric center of effort (GCE) of the sail plan. The wind speed and wind angle sensors were calibrated by wind tunnel tests in advance and the calibration equations were obtained. The Fujin also has motion measuring instruments such 
as an Optical Fiber Gyroscope (roll and pitch angles), a Flux Gate Compass (heading angle), a Differential type GPS receiver, a speedometer (velocity in the $x$ direction) and a potentiometer for rudder angle. These data are recorded by an onboard computer simultaneously with the data from the load cells.

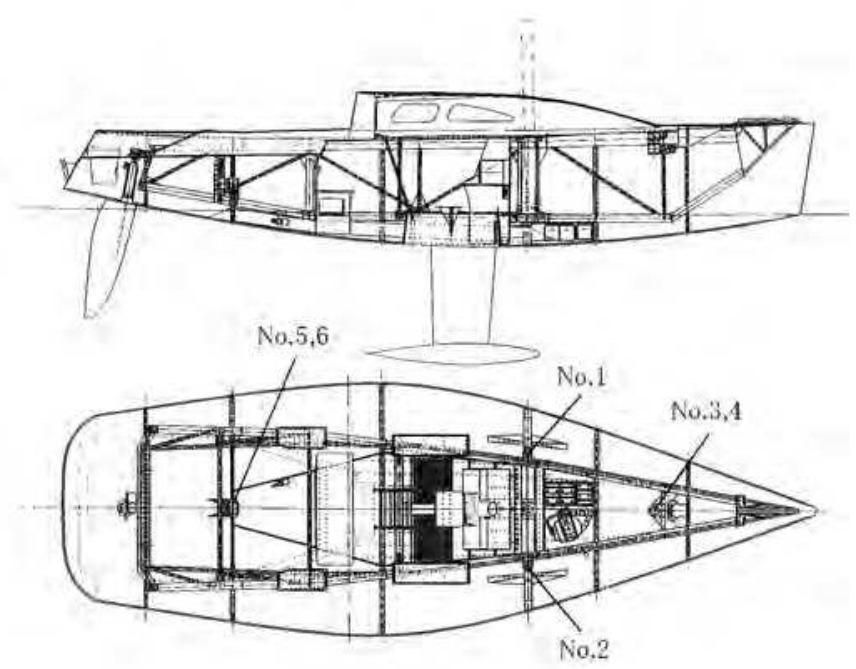

Fig. 10. General arrangement of dynamometer frame in Fujin.

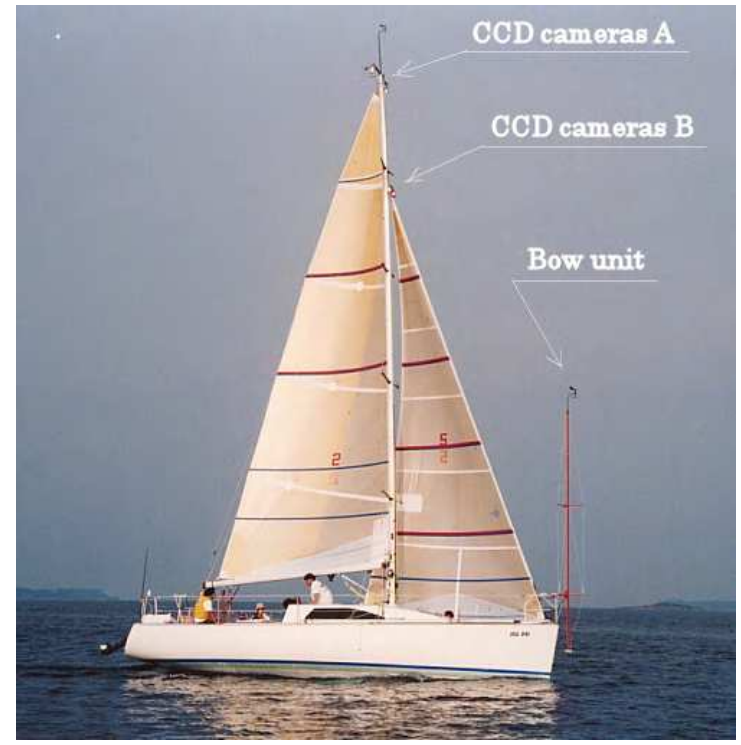

Fig. 11. Sea trial condition in light wind with $130 \%$ jib. 


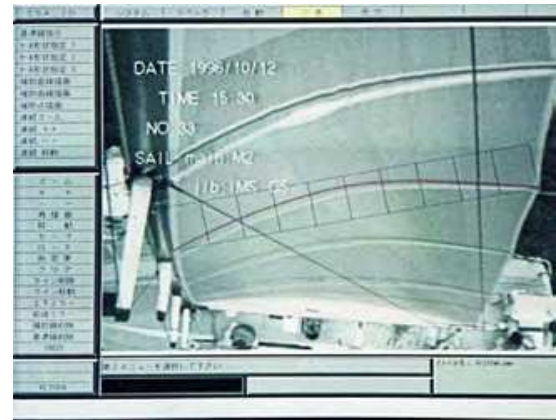

(a)

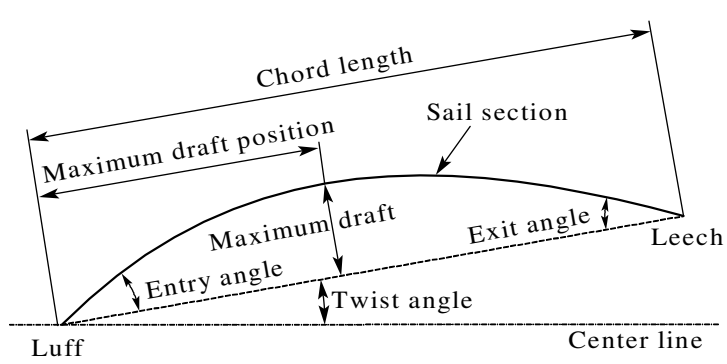

(b)

Fig. 12. (a) Example of a processed image of the mainsail using SSA-2D. (b) Measured sail shape parameters.

\subsubsection{Test condition and error analysis}

The sea tests were performed on Nanao Bay off the Noto Peninsula. The bay is approximately eight nautical miles from east to west and five from north to south. The bay is surrounded by low hills, and the mouth connecting it to the Japan Sea is narrow. Therefore, there is little tidal current in the bay, and the wave heights are relatively low even though the wind can be strong. The close-hauled tests were conducted over the apparent wind angle (AWA) range of 20 to 40 degrees, and the apparent wind speed (AWS) range of 5 to $11 \mathrm{~m} / \mathrm{s}$. The effect of the AWA, and the draft and twist of the mainsail on the sail performance were measured. Data sampling was started when the sailing condition was considered to be in steady state. The sampling rate for the data acquisition system was set at $10 \mathrm{~Hz}$. Data sampling was continued for 90 seconds, and during this time the sail shapes were recorded using the CCD cameras. The boat was steered carefully during this time. However, the measured data contained some variation due to wind fluctuation and wave reflection on the hull. Therefore the steady state values for the aerodynamic coefficients were obtained by averaging the data over a 30 to 60 seconds period, in which the AWA was closer to the target value than during the whole 90 second period. For these tests if the range of deviation of AWA exceeded \pm 5 degrees, the results were discarded. All of the measured coefficients are plotted with error bars indicating the range of deviation over the averaging period.

\subsection{Comparison between experimental and calculated results}

In this chapter, the experimental results and the calculated results for the following cases will be compared:

a. Variation with apparent wind angle

b. Variation with mainsail twist angle

For each series, first the sail coefficients: $C_{L}, C_{D}, C_{X}$ and $C_{Y}$, and the coordinates of $x_{C E}$ and $z_{C E}$ are given. Then, the calculated the sail surface pressure and streamlines using the RANSbased CFD are presented for two typical cases in each series. Finally, the shapes and threedimensional coordinates of the sails are tabulated for each case corresponding to those where the RANS-based CFD results are given. 


\subsubsection{Variation with apparent wind angle}

Fig.13 shows the performance variation for the mainsail and $130 \%$ jib configuration as a function of AWA. In the figure the solid symbols indicate the experimental results and the open symbols indicate the calculated results using the VLM and the RANS-based CFD. For the experimental results, both data from the starboard (Stbd) and port tack (Port) are shown. All of the measured coefficients are plotted with error bars indicating the range of deviation over the averaging period. There are some discrepancies between the data from each tack. During the experiments, efforts were made to remove this asymmetrical performance. However, the boat speed actually differed on each tack. It can be concluded that there was a slight asymmetry in the combination of the hull, keel, rudder and dynamometer frame. The numerical calculations were performed using the measured shape data. In order to avoid confusion when interpreting the figure, the calculated results are indicated only for the port tack. Therefore, the calculated and experimental points for the port tack correspond to each other.

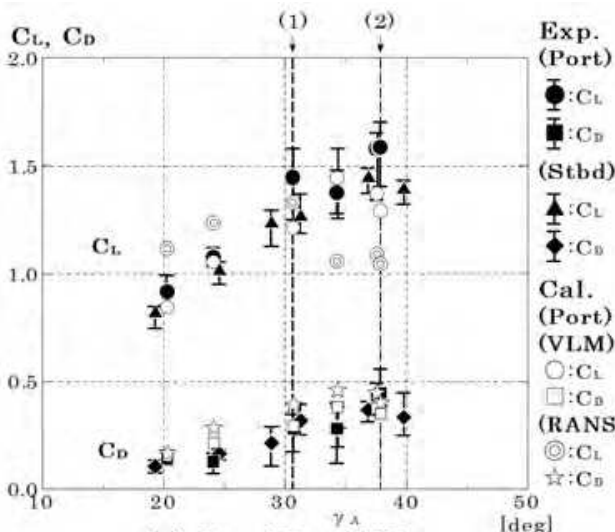

(a) CL and CD vs. AWA

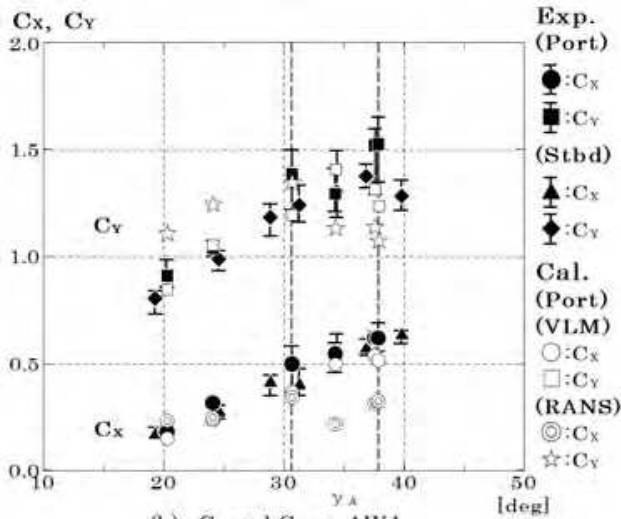

(b) $\mathrm{C}_{X}$ and $\mathrm{C}_{Y} \mathrm{vs}$. AWA

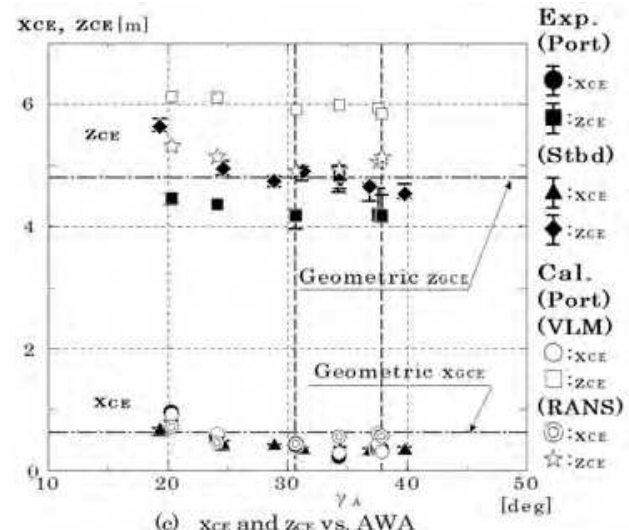

Fig. 13. Performance variation as a function of apparent wind angle (AWA) for mainsail and $130 \%$ jib. 
In this figure, AWA ranges from 20.3 degrees to 37.9 degrees for the port tack. The former is the closest angle to the wind that was achieved, and the latter is typical of a close reaching condition, where the sail is eased for the power down mode. There is some scatter in the experimental data because this is made up from measurements taken with the sails trimmed in slightly different ways. The experimental value of $C_{L}$ in Fig.13(a) varies with AWA from 0.91 to 1.58 . For the close reaching condition, the sails were not well trimmed to satisfy the power down mode. A sample of measured sail sections at this condition is shown in a figure attached to Table 2(2). From the figure, it can be seen that both the mainsail and the jib are not eased sufficiently to correspond to the large AWA. This is the reason for the decrement in the measured lift curve slope of $C_{L}$ at the range of AWA angles over about 35 degrees.

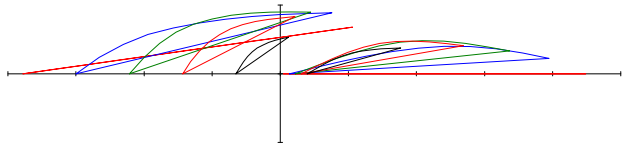

(1) 96092335

\begin{tabular}{|c|c|c|c|c|c|c|}
\hline & AWA deg & TWIST det & DRAFT $\Omega_{1}$ & AWS $(\mathrm{m} / \mathrm{s}]$ & HEEL(deB & VB $[\mathrm{kt}$ \\
\hline & 30.7 & 15.5 & 8.6 & 6.9 & 15.1 & 5.0 \\
\hline & $\overline{\mathrm{C}_{L}}$ & $\overline{c_{0}}$ & $\overline{C_{x}}$ & $\overline{C_{y}}$ & $x_{C F}[m]$ & $z_{O E}[m]$ \\
\hline & 1.44 & 0.28 & 0.50 & 1.39 & 0.41 & 4.17 \\
\hline 5 of & & $130 \% \mathrm{Jib}$ & & & Mainsail & \\
\hline & $x$ & $y$ & $z$ & $x$ & $y$ & 2 \\
\hline & -3.780 & 0000 & 0.000 & 0.046 & 0.000 & 1.320 \\
\hline & -2812 & 0136 & 0000 & 0.934 & 0.000 & 1.320 \\
\hline$\theta$ & -1.843 & 0.272 & 0.000 & 1.822 & 0.000 & 1.320 \\
\hline 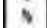 & -0.875 & 0.408 & 0.000 & 2.710 & 0.000 & 1.320 \\
\hline & 0.094 & 0.544 & 0.000 & 3.598 & 0.000 & 1.320 \\
\hline & 1.062 & 0.681 & 0.000 & 4.486 & 0.000 & 1.320 \\
\hline & -2998 & 0.000 & 2.140 & 0.133 & 0.000 & 3.820 \\
\hline & -2.305 & 0.429 & 2.140 & 0.888 & 0.176 & 3.820 \\
\hline 20 & -1.568 & 0.667 & 2.140 & 1.645 & 0.322 & 3.820 \\
\hline$s$ & -0.805 & 0.795 & 2.140 & 2.406 & 0.400 & 3.820 \\
\hline & -0.027 & 0.861 & 2.140 & 3.173 & 0.363 & 3.820 \\
\hline & 0.760 & 0.886 & 2.140 & 3.947 & 0.222 & 3.820 \\
\hline & -2.215 & 0.000 & 4.280 & 0.221 & 0.000 & 6,320 \\
\hline & -1.771 & 0.442 & 4.280 & 0.834 & 0.227 & 6.320 \\
\hline 40 & -1.272 & 0.719 & 4.280 & 1.452 & 0.405 & 6.320 \\
\hline 5 & -0.723 & 0.850 & 4.280 & 2.081 & 0.483 & 6.320 \\
\hline & $-0,145$ & 0.898 & 4.280 & 2.722 & 0.442 & 6.320 \\
\hline & 0.448 & 0.898 & 4.280 & 3.371 & 0.331 & 6.320 \\
\hline & -1433 & 0.000 & 6.420 & 0.308 & 0.000 & 8.820 \\
\hline & -1.486 & 0.332 & 6.420 & 0.761 & 0.218 & 8.820 \\
\hline 60 & -0.893 & 0.570 & 6.420 & 1.222 & 0.389 & 8.820 \\
\hline s & -0.552 & 0.715 & 6.420 & 1.699 & 0.470 & 8.820 \\
\hline & -0.176 & 0.790 & 6.420 & 2.191 & 0.462 & 8.820 \\
\hline & 0.217 & 0.832 & 6.420 & 2691 & 0.410 & 8.820 \\
\hline & -0.650 & 0.000 & 8.560 & 0.396 & 0.000 & 11,320 \\
\hline & -0.541 & 0.172 & 8.560 & 0.651 & 0.144 & 11.320 \\
\hline 80 & -0.414 & 0.318 & 8.560 & 0.914 & 0.261 & 11.320 \\
\hline 4 & -0.255 & 0.419 & 8.560 & 1.190 & 0.330 & 11.320 \\
\hline & -0.073 & 0.486 & 8.560 & 1.476 & 0.362 & 11320 \\
\hline & 0.122 & 0.535 & 8.560 & 1768 & 0.374 & 11320 \\
\hline & 0.132 & 0000 & 10.700 & 0.483 & 0.000 & 13.820 \\
\hline & 0.144 & 0.016 & 10.700 & 0.511 & 0.012 & 13.820 \\
\hline 100 & 0.159 & 0.030 & 10,700 & 0.538 & 0.023 & 13.820 \\
\hline 5 & 0.173 & 0.044 & 10.700 & 0.567 & 0.033 & 13.820 \\
\hline & 0.189 & 0.056 & 10.700 & 0.595 & 0.042 & 13.820 \\
\hline & 0.207 & 0.066 & 10.700 & 0.624 & 0.051 & 13.820 \\
\hline
\end{tabular}

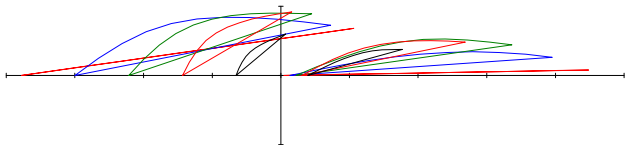

(2) 96080248

\begin{tabular}{|c|c|c|c|c|c|c|}
\hline & AWA deg & \multicolumn{2}{|c|}{ TWIST deADRAFTIS } & \multicolumn{2}{|c|}{ AWS/m/s HEELIdeg. } & VB $(\mathrm{kt})$ \\
\hline & 37.9 & 14.5 & 7.2 & \multicolumn{2}{|c|}{\begin{tabular}{r|r|}
7.5 & 19.6 \\
\end{tabular}} & 6.0 \\
\hline & $\mathrm{C}_{2}$ & $\mathrm{C}_{\mathrm{b}}$ & $\mathrm{C}_{\mathrm{x}}$ & $\mathrm{C}_{y}$ & $x_{e E[}[m]$ & $\mathrm{z}_{\mathrm{CE}}[\mathrm{m}]$ \\
\hline & 1.58 & 0.45 & 0.62 & 1.52 & 0.34 & 4.17 \\
\hline \multirow{2}{*}{$\begin{array}{l}\text { S of } \\
\text { heit }\end{array}$} & \multicolumn{3}{|c|}{$130 \%$ Jib } & \multicolumn{3}{|c|}{ Mainsail } \\
\hline & \multicolumn{2}{|l|}{$x$} & 2 & $x$ & $y$ & $z$ \\
\hline \multirow{6}{*}{$\begin{array}{l}0 \\
3\end{array}$} & -3.780 & 0,000 & 0.000 & 0,046 & 0.000 & 1.320 \\
\hline & -2.812 & 0.136 & 0,000 & 0.934 & 0.015 & 1.320 \\
\hline & -1.843 & 0.272 & 0.000 & 1.822 & 0.031 & 1.320 \\
\hline & -0.875 & 0.408 & 0.000 & 2.710 & 0.046 & 1.320 \\
\hline & 0.094 & 0.544 & 0.000 & 3.597 & 0.062 & 1.320 \\
\hline & 1.062 & 0.681 & 0.000 & 4.485 & 0.077 & 1.320 \\
\hline \multirow{6}{*}{$\begin{array}{c}20 \\
8\end{array}$} & $-2,998$ & 0,000 & 2,140 & 0.133 & 0.000 & 3.820 \\
\hline & -2.314 & 0.461 & 2.140 & 0.891 & 0.150 & 3.820 \\
\hline & -1.597 & 0,750 & 2,140 & 1.651 & 0.267 & 3.820 \\
\hline & -0.841 & 0.840 & 2.140 & 2.414 & 0.331 & 3.820 \\
\hline & -0.062 & 0.810 & 2.140 & 3.182 & 0.333 & 3.820 \\
\hline & 0.728 & 0.724 & 2.140 & 3.954 & 0.262 & 3.820 \\
\hline \multirow{6}{*}{$\begin{array}{c}40 \\
5\end{array}$} & -2.215 & 0.000 & 4.280 & 0.221 & 0.000 & 6320 \\
\hline & -1.769 & 0.437 & 4.280 & 0.829 & 0.239 & 6.320 \\
\hline & -1.274 & 0.729 & 4.280 & 1.445 & 0.423 & 6.320 \\
\hline & -0.726 & 0.863 & A.280 & 2.074 & 0.520 & 6. 320 \\
\hline & -0.145 & 0.899 & 4.280 & 2717 & 0.511 & 6.320 \\
\hline & 0.450 & 0.892 & 4.280 & 3.368 & 0.442 & 6.320 \\
\hline \multirow{6}{*}{$\begin{array}{c}60 \\
5\end{array}$} & -1.433 & 0,000 & 6.420 & 0.308 & 0.000 & 8.820 \\
\hline & -1.218 & 0.362 & 6.420 & 0.757 & 0.230 & 8.820 \\
\hline & -0.940 & 0.615 & 6.420 & 1.218 & 0.397 & 8.820 \\
\hline & -0.601 & 0.763 & 6.420 & 1.697 & 0.482 & 8.820 \\
\hline & -0.230 & 0.854 & 6.420 & 2.187 & 0.504 & 8.820 \\
\hline & 0.157 & 0.918 & 6.420 & 2687 & 0.481 & B.820 \\
\hline \multirow{6}{*}{$\begin{array}{c}80 \\
5\end{array}$} & -0.650 & 0.000 & 8560 & 0,396 & 0.000 & 11.320 \\
\hline & -0.565 & 0.191 & 8.560 & 0.656 & 0.128 & 11.320 \\
\hline & -0.445 & 0.339 & 8.560 & 0.921 & 0.241 & 11.320 \\
\hline & -0.289 & 0.444 & 8.560 & 1.193 & 0.327 & 11.320 \\
\hline & -0.113 & 0.527 & 8560 & 1.478 & 0.368 & 11.320 \\
\hline & 0.071 & 0.597 & 8.560 & 1.771 & 0.377 & 11.320 \\
\hline \multirow{6}{*}{$\begin{array}{c}100 \\
5\end{array}$} & 0.132 & 0.000 & 10,700 & 0.483 & 0.000 & 13.820 \\
\hline & 0.142 & 0.018 & 10,700 & 0.511 & 0.011 & 13.820 \\
\hline & 0.154 & 0.034 & 10,700 & 0.539 & 0.022 & 13.820 \\
\hline & 0.167 & 0.049 & 10.700 & 0.567 & 0.032 & 13.820 \\
\hline & 0.181 & 0064 & 10.700 & 0.596 & 0.041 & 13.820 \\
\hline & 0.196 & 0.077 & 10.700 & 0.625 & 0.049 & 13.820 \\
\hline
\end{tabular}

Table 2. Sail shapes, measured experimental data and three-dimensional coordinates of the sails for the cases of (1) 96092335 and (2) 96080248. 
The calculated results for $C_{L}$ using the VLM show good agreement with the experiments at AWA angles less than about 35 degrees. Over about 35 degrees, the calculated results are lower than the measured ones. This shows that the calculated results strongly indicate the effect of incorrect sail trimming. The results for $C_{L}$ using the RANS-based CFD show the same trends with the experiments, but are slight higher than those from the experiments for AWA between 20 degrees to 30 degrees and lower for AWA greater than 30 degrees. In particular, the decrease in $C_{L}$ for AWA values greater than 30 degrees is considerably large. This will be discussed later with the calculated sail surface pressure and streamlines. The calculated results for $C_{D}$ slightly over predict those from the experiments. Fig.13(c) shows the coordinates of the center of effort of the sails. The $x$ and $z$ coordinates of the geometric center of effort $\left(x_{G C E}\right.$ and $z_{G C E}$ ) are $0.63 \mathrm{~m}$ aft and $4.80 \mathrm{~m}$ above the origin, which are indicated by alternate long and short dashed lines in the figure. It is seen that both the experimental and the calculated coordinates of $x_{C E}$ are near $x_{G C E}$ and move slightly forward with increasing AWA. Unfortunately, there is a wide scatter in the experimental values of $z_{C E}$. This is thought to be because the measured Ks moment contains a large component from the mass of the dynamometer frame and rigging $(659 \mathrm{~kg})$. This moment was subtracted from the measurement, taking into account the measured heel angle. If there is a slight error in the position of center of gravity of the dynamometer frame, or in the measured heel angle, the error in the calculated moment will be large. However, though there is a scatter in the measured data, it can be seen that $z_{C E}$ is decreasing as AWA increases. The trends in the movement of both $x_{C E}$ and $z_{C E}$ as functions of AWA might be caused by the decrement of force acting on the aft and upper parts of the sails due to the loosening of main and jib sheets with increasing AWA. The calculated results for $z_{C E}$ obtained using theRANS-based CFD show the same trend as the experiments. On the other hand, the calculated results using VLM are considerably higher than the experimental ones. This might be caused by over estimation of the force acting on the upper portion of the mainsail. In this area, since the jib is not overlapping, flow separation may occur easily. However, the VLM does not take flow separation into account.

Figures 14(1) and 14(2) show the calculated results of the sail surface pressure and streamlines using RANS- based CFD. Fig.14(1) indicates the case of experiment ID 96092335 (AWA= 30.7deg.), and 14(2) indicates ID 96080248 (AWA= 37.9deg.). These data correspond to the plotted points on the vertical dotted lines (1) and (2) in Fig.13. In Fig.14, the left and right diagrams correspond to the port and starboard sides, i.e., pressure and suction sides, respectively. In 14(1), although slight flow separation on the suction side of mainsail is seen, the streamlines of both sides run smoothly. On the other hand, in 14(2), considerable flow separation is occurring, in particular, on the suction side of jib. This is the main reason for the reduction of $C_{L}$ value in the RANS-based CFD calculation at (2) in Fig.13(a). The shapes and three-dimensional coordinates of the sails are given in Table 2. The numbered (1) and (2) tables correspond to the cases of experiment ID 96092335 and ID 96080248, respectively. These also correspond to the calculated results shown in Fig.14. The figures described above the tables show the sail section profiles at $0,20,40,60$ and $80 \%$ of the sail height. The dimensions of these three-dimensional coordinates are given in the tables including $100 \%$ height section data. The positive direction of the $x$ coordinate is aft. The four lines at the top of the tables are the measured values for the wind and sail trim conditions, the boat attitude and the sail performance coefficients. 
(1)
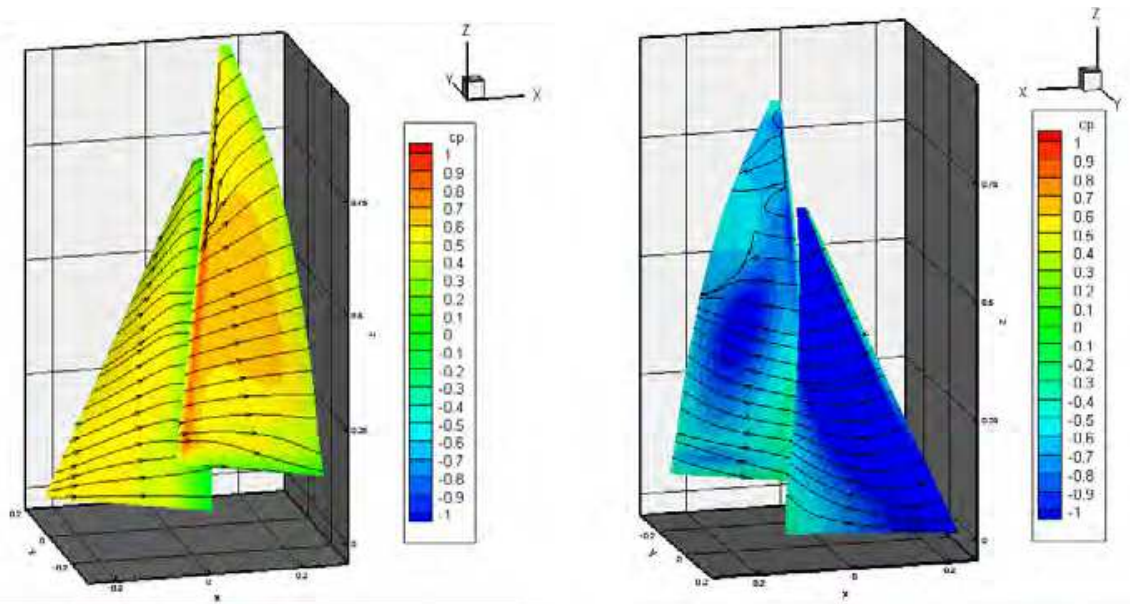

(2)
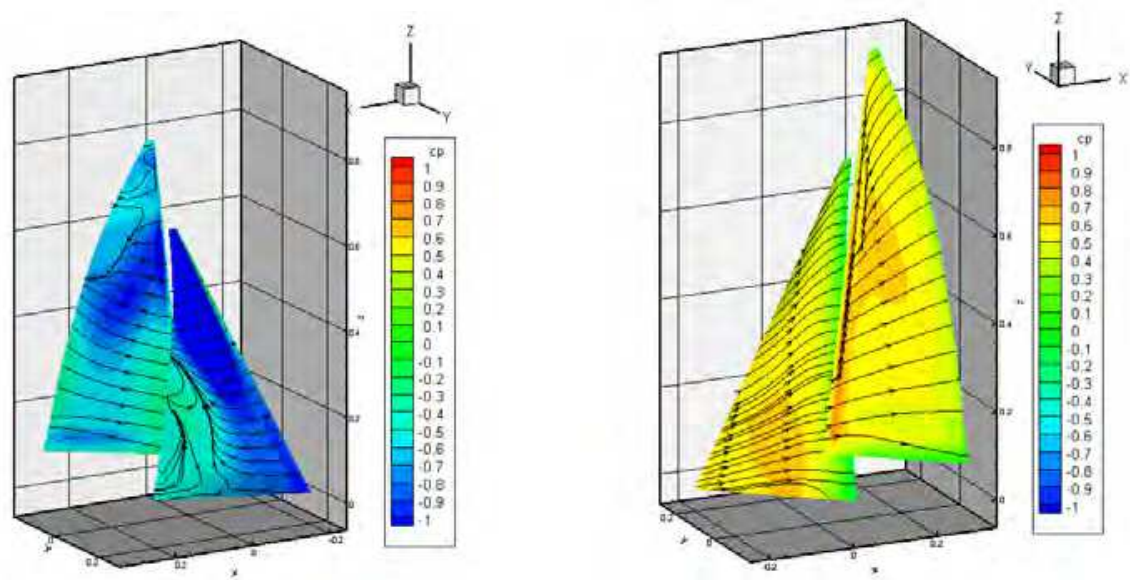

Fig. 14. (1) Surface pressure and streamlines obtained by RANS-based CFD at experimental ID 96092335 (AWA=30.7 deg.) and (2) ID 96080248 (AWA=37.9 deg.).

\subsubsection{Variation with mainsail twist angle}

Fig.15 shows the performance variation for the mainsail and $130 \%$ jib configuration as a function of mainsail twist angle. The mainsail twist was changed by varying the main sheet tension. The boom angle was kept parallel with the boat centerline by moving the main sheet traveler. The experiment was performed for an average value of AWA of $30 \pm 2$ degrees and mean draft at around $10 \%$. The jib shape was fixed. The twist angle is defined as the angle between the boom line and section chord line at $80 \%$ height. In the figure, the 
twist angle ranges from 4.5 degrees to 24.9 degrees for the port tack. Varying the twist angle by 20.4 degrees, results in the value of $C_{X}$ in Fig. 15(b) changing from 0.33 to 0.39 (18\%), and the value of $C_{Y}$ changing from 1.16 to $1.39(20 \%)$. It can be seen that the maximum $C_{X}$ occurs at a twist angle of around 15 degrees. The considerable decrease in $C_{Y}$ with increasing twist angle is also worth noticing. In this case, the calculated results of both VLM and RANSbased CFD for $C_{X}$ and $C_{Y}$, and $C_{L}$ and $C_{D}$ correspond to the measured values very well.

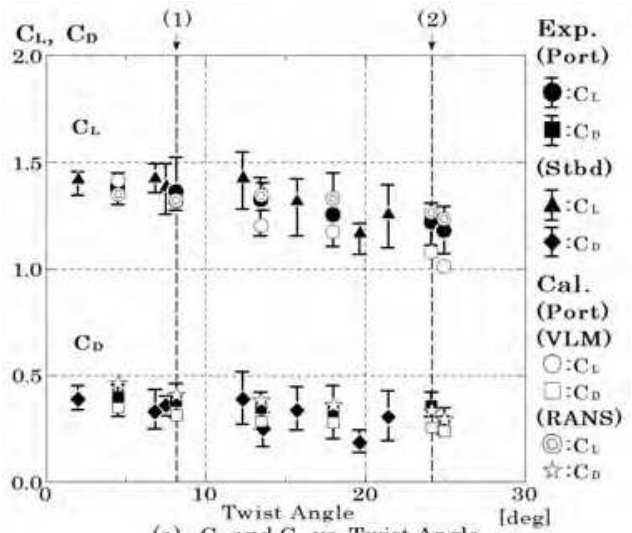

(a) Ctand Co vos. Twist Angle

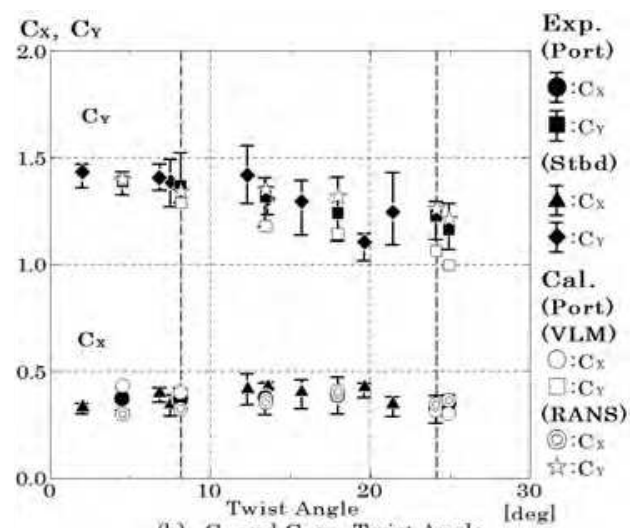

(b) $\mathrm{C}_{\mathrm{x}}$ and $\mathrm{C}_{\mathrm{r}} \mathrm{vs}$, Twist Angle

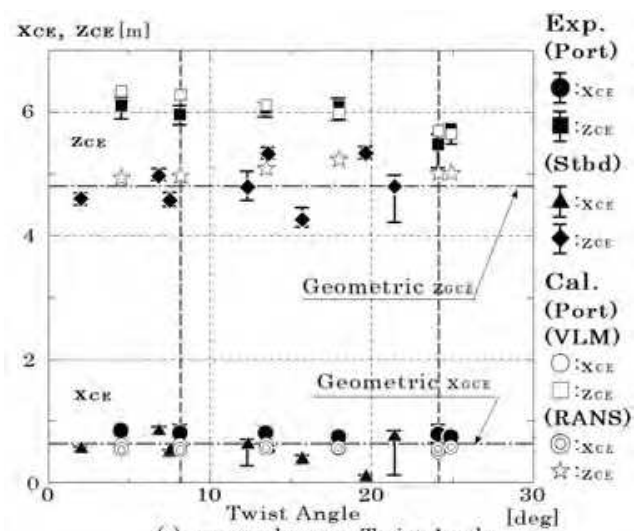

(c) XCE and zce vs. Twist Angle

Fig. 15. Performance variation as a function of mainsail twist angle for mainsail and $130 \%$ jib.

Figures 16(1) and 16(2) show the calculated results using RANS-based CFD. Fig.16(1) corresponds to ID 97072213 (twist angle $=8.2$ deg.), and 16(2) corresponds to ID 97072218 (twist angle $=24.1$ deg.). It can be seen in Fig.16(1) that the streamlines on the upper part of the suction side of the mainsail for the smaller twist angle, show considerable flow 
separation. This is caused by the large angle of attack at the upper part of the sail due to the small twist angle. On the other hand, for the higher twist angle shown in Fig.16(2), there is a low negative pressure area at the luff on the suction side of mainsail due to the small angle of attack. This is what causes the considerable reduction in the calculated value for $C_{X}$ at (2) in Fig.15(b). Table 3 shows the shapes and three-dimensional coordinates of the sails for cases (1) and (2), which correspond to the calculated results shown in Fig.16. Further measured data and comparison with the numerical calculations are described by Masuyama et al. (2007 and 2009)

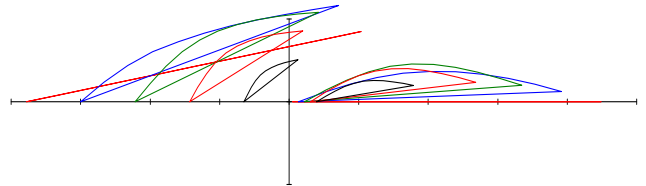

(1) 97072213

\begin{tabular}{|c|c|c|c|c|c|c|}
\hline & AWA des] & TWIST de & DRAFT[9] & \multicolumn{2}{|c|}{ AWS $\mathrm{m} / \mathrm{s}$ HEEL/deg } & VB $[\mathrm{kt}]$ \\
\hline & 30.7 & 8.2 & 10.5 & 73 & 16.8 & 5.1 \\
\hline & $\overline{\mathrm{C}_{\mathrm{i}}}$ & $\overline{\mathrm{C}_{0}}$ & $\overline{C_{x}}$ & $\overline{\mathrm{C}_{r}}$ & $x_{c:}[m]$ & $z_{C E}[m]$ \\
\hline & 1.36 & 0.38 & 0.37 & 1.37 & 0.79 & 5.96 \\
\hline \multirow{2}{*}{$\begin{array}{l}\text { \% of } \\
\text { heit }\end{array}$} & \multicolumn{3}{|c|}{$130 \% \mathrm{Jib}$} & \multicolumn{3}{|c|}{ Mainsail } \\
\hline & \multicolumn{2}{|r|}{$y$} & $z$ & \multicolumn{2}{|l|}{$x$} & 2 \\
\hline \multirow{6}{*}{$\begin{array}{l}0 \\
5\end{array}$} & -3.780 & 0.000 & 0.000 & 0046 & 0.000 & 1,320 \\
\hline & -2.817 & 0.170 & 0,000 & 0.934 & 0.000 & 1.320 \\
\hline & -1.854 & 0.340 & 0.000 & 1.822 & 0.000 & 1.320 \\
\hline & -0.891 & 0.509 & 0.000 & 2710 & 0.000 & 1.320 \\
\hline & 0.073 & 0.679 & 0.000 & 3.598 & 0.000 & 1.320 \\
\hline & 1.036 & 0.849 & 0.000 & 4.486 & 0.000 & 1.320 \\
\hline \multirow{6}{*}{$\begin{array}{c}20 \\
5\end{array}$} & -2.998 & 0.000 & 2.140 & 0.133 & 0.000 & 3.820 \\
\hline & -2.320 & 0.440 & 2.140 & 0.884 & 0.214 & 3.820 \\
\hline & -1.595 & 0.727 & 2.140 & 1.638 & 0.345 & 3.820 \\
\hline & -0.839 & 0.917 & 2.140 & 2.395 & 0.362 & 3.820 \\
\hline & -0.067 & 1055 & 2.140 & 3.156 & 0.283 & 3820 \\
\hline & 0.714 & 1.165 & 2140 & 3.918 & 0.125 & 3.820 \\
\hline \multirow{6}{*}{$\begin{array}{c}40 \\
5\end{array}$} & -2.215 & 0,000 & 4.280 & 0.221 & 0.000 & 6.320 \\
\hline & -1.774 & 0.427 & 4.280 & 0.832 & 0.263 & 6.320 \\
\hline & -1289 & 0.746 & -4.280 & 1.449 & 0.427 & 6.320 \\
\hline & -0.750 & 0.933 & 4.280 & 2.076 & 0.452 & 6.320 \\
\hline & -0.174 & 1.031 & 4.280 & 2710 & 0.358 & 6.320 \\
\hline & 0.421 & 1.082 & 4.280 & 3.348 & 0.199 & 6.320 \\
\hline \multirow{6}{*}{60} & -1.433 & 0.000 & 6.420 & $0.30 B$ & 0.000 & 8.820 \\
\hline & -1.193 & 0.336 & 6.420 & 0.766 & 0.231 & 8.820 \\
\hline & -0.911 & 0.590 & 6.420 & 1.232 & 0.372 & 8.820 \\
\hline & -0.573 & 0.737 & 6.420 & 1.710 & 0.399 & 8.820 \\
\hline & -0.199 & 0.816 & 6.420 & 2.196 & 0.340 & 8.820 \\
\hline & 0.196 & 0.855 & 6420 & 2.687 & 0.235 & 8.820 \\
\hline \multirow{6}{*}{$\begin{array}{c}80 \\
\%\end{array}$} & -0.650 & 0.000 & 8.560 & 0.396 & 0.000 & 11.320 \\
\hline & -0.543 & 0.176 & 8.560 & 0.662 & 0.130 & 11,320 \\
\hline & -0.416 & 0.322 & 8.560 & 0.932 & 0.224 & 11.320 \\
\hline & -0.255 & 0.414 & 8.560 & 1.212 & 0.259 & 11.320 \\
\hline & -0.070 & 0.472 & B.560 & 1.499 & 0.241 & 11.320 \\
\hline & 0.128 & 0.510 & 8.560 & 1.789 & 0.197 & 11.320 \\
\hline \multirow{6}{*}{$\begin{array}{c}100 \\
\leqslant\end{array}$} & 0.132 & 0.000 & 10.700 & 0.483 & 0.000 & 13,820 \\
\hline & 0.145 & 0.016 & 10.700 & 0.512 & 0.007 & 13.820 \\
\hline & 0.161 & 0.028 & 10,700 & 0.541 & 0.014 & 13.820 \\
\hline & 0.177 & 0.041 & 10.700 & 0.577 & 0.019 & 13.820 \\
\hline & 0.193 & 0.052 & 10.700 & 0.601 & 0.023 & 13.820 \\
\hline & 0.212 & 0.060 & 10.700 & 0.630 & 0.027 & 13.820 \\
\hline
\end{tabular}

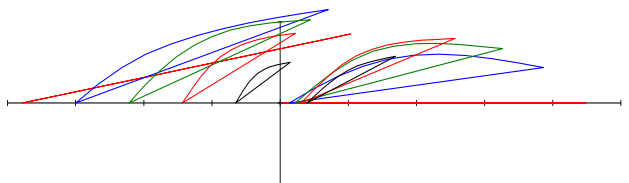

(2) 97072218

\begin{tabular}{|c|c|c|c|c|c|c|}
\hline & AWA deg & TWIST de & RAFT $(S)$ & AWS $(m / s)$ & HEEL(deg) & VB [kt] \\
\hline & 31.1 & 24.1 & 10.6 & 7.2 & 12.3 & 5.1 \\
\hline & $\mathrm{C}_{2}$ & $\mathrm{C}_{0}$ & $C_{x}$ & $\mathrm{C}_{\mathrm{y}}$ & $x_{c F}[m]$ & $z_{\text {OE }}[m]$ \\
\hline & 1.22 & 0.36 & 0.33 & 1.23 & 0.78 & 5.47 \\
\hline \multirow{2}{*}{$\begin{array}{l}\% \text { af } \\
\text { heit }\end{array}$} & \multicolumn{3}{|c|}{$130 \% \mathrm{Jib}$} & \multicolumn{3}{|c|}{ Mainsail } \\
\hline & $x$ & $y$ & 2 & $x$ & $y$ & 2 \\
\hline \multirow{6}{*}{$\begin{array}{l}0 \\
3\end{array}$} & -3.780 & 0000 & 0.000 & 0.046 & 0.000 & 1.320 \\
\hline & -2.817 & 0.170 & 0.000 & 0.934 & 0.000 & 1.320 \\
\hline & -1.854 & 0.340 & 0.000 & 1,822 & 0.000 & 1.320 \\
\hline & -0.891 & 0.509 & 0.000 & 2710 & 0.000 & 1.320 \\
\hline & 0.073 & 0.679 & 0.000 & $3.59 \mathrm{~B}$ & 0.000 & 1320 \\
\hline & 1.036 & 0.849 & 0.000 & 4.486 & 0.000 & 1.320 \\
\hline \multirow{6}{*}{$\begin{array}{c}20 \\
5\end{array}$} & -2.988 & 0.000 & 2.140 & 0.133 & 0.000 & 3.820 \\
\hline & -2.321 & 0.439 & 2.140 & 0.852 & 0.328 & 3.820 \\
\hline & -1.598 & 0.728 & 2.140 & 1.584 & 0.539 & 3.820 \\
\hline & -0.844 & 0,914 & 2.140 & 2334 & 0.601 & 3.820 \\
\hline & -0.073 & 1048 & 2140 & 3.097 & 0.548 & 3,820 \\
\hline & 0.708 & 1.151 & 2140 & 3.866 & 0.437 & 3.820 \\
\hline \multirow{6}{*}{$\begin{array}{c}40 \\
5\end{array}$} & -2.215 & 0.000 & 4.280 & 0.221 & 0.000 & 6.320 \\
\hline & -1.760 & 0.403 & 4.280 & 0.777 & 0.378 & 6.320 \\
\hline & -1.266 & 0,707 & 4.280 & 1.358 & 0.638 & 6.320 \\
\hline & -0.729 & 0.898 & 4.280 & 1.977 & 0.730 & 6.320 \\
\hline & -0.151 & 0.985 & 4.280 & 2.617 & 0.721 & 6.320 \\
\hline & 0.444 & 1.025 & 4.280 & 3.268 & 0.669 & 6.320 \\
\hline \multirow{6}{*}{$\begin{array}{l}60 \\
5\end{array}$} & -1.433 & 0.000 & 6.420 & $0.30 B$ & 0.000 & 8.820 \\
\hline & -1.173 & 0.313 & 6.420 & 0.701 & 0.328 & 8.820 \\
\hline & -0.883 & 0.565 & 6.420 & 1.119 & 0.582 & 8.820 \\
\hline & -0.544 & 0.724 & 6.420 & 1.580 & 0.716 & 8.820 \\
\hline & -0.168 & 0.811 & 6.420 & 2.068 & 0.771 & 8.820 \\
\hline & 0.229 & 0.858 & 6.420 & 2.568 & 0.794 & 8.820 \\
\hline \multirow{6}{*}{$\begin{array}{l}80 \\
\%\end{array}$} & -0.650 & 0000 & 8.560 & 0.396 & 0.000 & 11.320 \\
\hline & -0.533 & 0.166 & 8.560 & 0.627 & 0.179 & 11.320 \\
\hline & -0.401 & 0.310 & 8.560 & 0.868 & 0.335 & 11.320 \\
\hline & -0.243 & 0.411 & 8.560 & 1.127 & 0.453 & 11.320 \\
\hline & -0.056 & 0.467 & 8.560 & 1.406 & 0.525 & 11.320 \\
\hline & 0.146 & D.500 & B. 560 & 1.694 & 0.576 & 11.320 \\
\hline \multirow{6}{*}{$\begin{array}{c}100 \\
7\end{array}$} & 0.132 & 0000 & 10.700 & 0.483 & 0.000 & 13.820 \\
\hline & 0.145 & 0.016 & 10.700 & 0.509 & 0.015 & 13.820 \\
\hline & 0.161 & 0.028 & 10.700 & 0.535 & 0.031 & 13.820 \\
\hline & 0.177 & 0.040 & 10.700 & 0.561 & 0.045 & 13.820 \\
\hline & 0.193 & 0.052 & 10.700 & 0.588 & 0.057 & 13.820 \\
\hline & 0.212 & 0.060 & 10.700 & 0.615 & 0.070 & 13.820 \\
\hline
\end{tabular}

Table 3. Sail shapes, measured experimental data and three-dimensional coordinates of the sails for the cases of (1) 97072213 and (2) 97072218. 
(1)
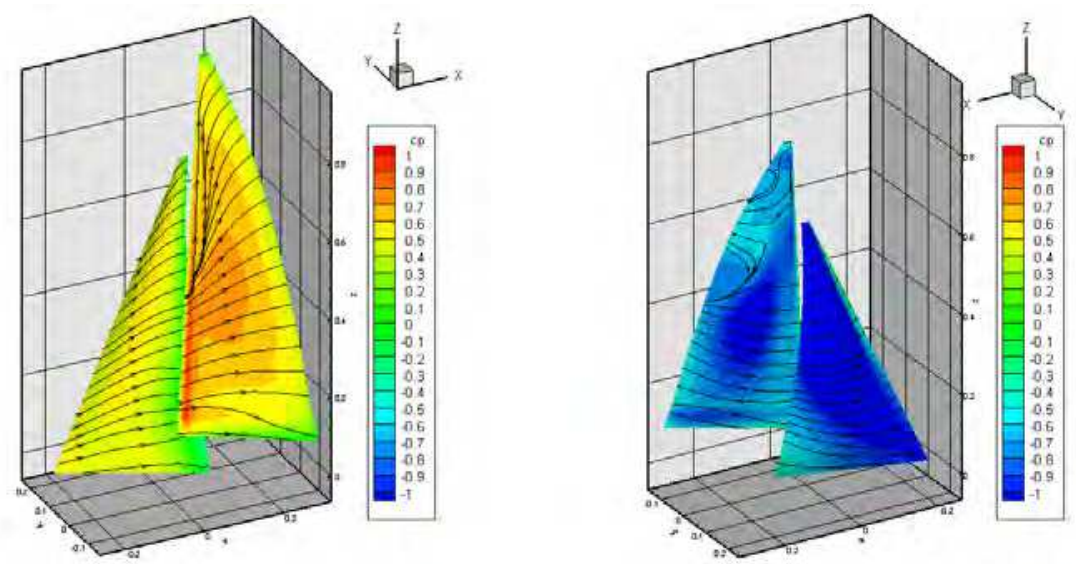

(2)
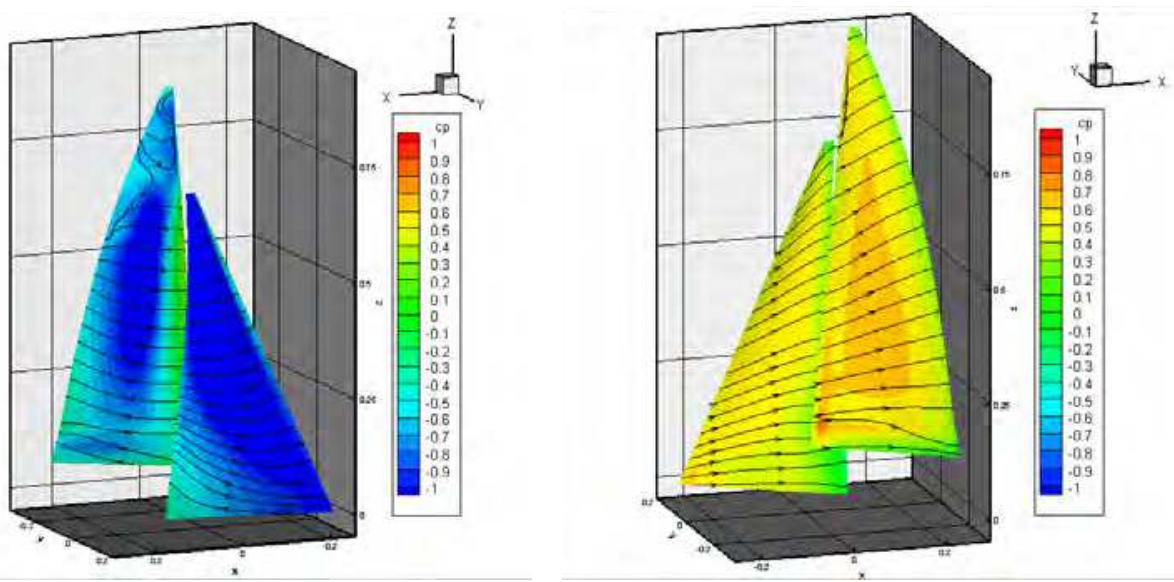

Fig. 16. (1) Surface pressure and streamlines obtained by RANS-based CFD at experimental ID 97072213 (twist angle= 8.2 deg.) and (2) ID 97072218 (twist angle= 24.1 deg.).

\section{Discussion of numerical calculation methods}

The flow is dominated by multiple-lifting-surface aerodynamic interactions. For larger AWA values, in particular, a large-scale flow separation exists on the leeward side of the sails. In general, there is complex vortex generation in the wake, especially near the top and bottom of the sails, i.e., tip vortices are generated and are influenced by the boundary layer flows on the sails. The resultant aerodynamic forces are mostly dominated by the pressure component, whereas the contribution of the frictional component is generally small. The accurate prediction of the boundary layer flows on the sails and the three-dimensional flow separation, associated with the abovementioned vortex generation, are big challenges for 
RANS-based CFD. The geometrical complexity is also another significant challenge to RANS-based CFD. The accuracy in the prediction of the CE is of great interest, in association with the correct prediction of the above-mentioned three-dimensional flow separation.

Through the analyses of the multiblock RANS-based CFD, it appears that the overall trends of the flow and the aerodynamic forces measured in the experiments are fairly well predicted by the present computations. It is also seen that the multiblock domain decomposition considered here is very effective for the present mainsail and jib configurations. The automatic gridding scheme used successfully generates high-quality structured grids for the various sail geometries, AWA, and heel angles considered in the present study. Although there are advantages to a structured grid system for highresolution in the boundary layer flow, building a grid in this fashion is difficult to apply to complex geometries. This problem appears to be resolved by the present scheme.

The Vortex Lattice method is, on the other hand, a convenient tool to predict the lift and induced drag acting on the sail accurately at the apparent wind angles less than about 35 degrees. The computational time of the method is about a few minutes for one calculation condition. The longitudinal coordinates, or $x$ coordinate, of the center of effort of the sail can also be calculated with accuracy by the Vortex Lattice method, however, the estimated vertical coordinate, or z coordinate, of the center of effort by the Vortex Lattice method is considerably higher than the experimental ones. This may be caused by the fact that the flow at the upper portion of mailsail is easily separated because of the absence of jib overlapping, while the flow separation cannot be taken into account in the Vortex Lattice method

\section{Conclusion}

The sail performance analysis of sailing yacht was carried out by using numerical calculations and experiments. Focus in the present manuscript is especially on the upwind sailing condition. The sails considered here are IMS type, and the shapes and performance were measured by using the sail dynamometer boat Fujin. The measured sail flying shapes were used by numerical analysis, where two CFD methods developed by the authors were used, i.e. a multiblock RANS-based CFD method by Tahara and a VLM-based CFD method by Fukasawa. It appears that the overall trends of the flow and the aerodynamic forces measured in the experiments are fairly well predicted by the present computations; and at the same time, the present sail performance database based on the full scale onboard measurements are very useful for validation study of numerical methods. As compared to maturity of VLM, that for RANS-based CFD is still in underway but the future prospect is shown promising, especially for capability in predicting separation flow filed where viscous effects of fluid are significant. The authors believe that our sail performance database associated with accurate sail flying shape measurements will be able to contribute to the further development of more advanced CFD methods.

Although details are not described in the present manuscript, our current effort is directed toward the more challenging problem, i.e., extension of the present work for the downwind sailing condition. Since the onboard sail shape measurement system of Fujin is incompetent for the spinnaker measurement due to its balloon shape, the sail shapes and performance are measured using wind tunnel equipment, and such activities are already in progress. The sail shapes are recorded using digital cameras and processed to obtain 3D coordinates using solid shape analyzer software, which provides 3D coordinates from digital photographs taken from several different directions. Importantly, the sail shapes and the sail forces and 
moment acting on the model are measured simultaneously. The numerical simulation by using a RANS-based CFD method is also in progress. Along with integration with the aforementioned sail design and performance prediction software $A A F$, more details of our work will be reported in our future publications.

\section{Acknowledgments}

The sail dynamometer boat Fujin was built for sail tests for the Japanese America's Cup entry by a Grant-in-Aid from the Nippon Foundation and the authors would like to thank the Nippon Foundation for providing them this invaluable tool. The authors wish to express their thanks to Yamaha Motor Co. Ltd. for building Fujin and to North Sails Japan Co. for making the sails. We would like to thank Dr. Martin Renilson for his valuable discussions and comments on this article. We would also like to thank Mr. H. Mitsui, the harbormaster of the Anamizu Bay Seminar House of the Kanazawa Institute of Technology, for his assistance with the sea trials. Help with the sea trials given by graduate and undergraduate students of the Kanazawa Institute of Technology is also gratefully acknowledged. The graduate students were Masaya Miyagawa, Takashi Hasegawa, and Munehiko Ogihara. Finally, we would like to acknowledge the effort by Mr. Naotoshi Maeda who carried out most of the RANS-CFD simulations as a part of his work on M.S. thesis at Osaka Prefecture University.

\section{References}

Baldwin, B.S. \& Lomax, H. (1978). Thin Layer Approximation and Algebraic Model for Separated Turbulent Flows, AIAA Paper, 78-257, AIAA 16th Aerospace Science Meeting, Reno, NV., USA, 1978

Falkner, V.M. (1943). The Calculation of Aerodynamic Loading on Surface of Any Shape, Aeronautical Research Committee Reports and Memoranda No.1910, Ministry of Aircraft Production, London, UK

Falkner, V.M. (1946). The Accuracy of Calculations Based on Vortex Lattice Theory, British Aeronautical Research Council, No.9621

Fukasawa, T. \& Katori, M. (1993). Numerical Approach to Aeroelastic Responses of ThreeDimensional Flexible Sails, Proceedings of 11th Chesapeake Sailing Yacht Symposium, pp.87-105, Annapolis, USA, January 29-30, 1993

Fukasawa, T. (1993). Aeroelastic Transient Response of 3-Dimensional Flexible Sail, Proceedings of International Conference on Aero-Hydroelasticity, pp.57-62, Beijing, China, October 18-21, 1993,

Hansen, H., Jackson, P. \& Hochkirch, K. (2003). Comparison of Wind Tunnel and Full-scale Aerodynamic Sail Force, International Journal of Small Craft Technology (IJSCT), Vol. 145 Part B1, (2003), pp. 23-31

Hochkirch, K. \& Brandt, H. (1999). Fullscale Hydrodynamic Force Measurement on the Berlin Sailing Dynamometer, Proceedings of SNAME 14th Chesapeake Sailing Yacht Symposium, pp.33-44, Annapolis, Maryland, USA, January, 1999

Katori, M. (2009). Advanced Aero Flow Software Manual, North Sails Japan, December 2009.

Krebber, B. \& Hochkirch, K. (2006). Numerical Investigation on the Effects of Trim for a Yacht Rig, Proceedings of 2nd High Performance Yacht Design Conference, pp.13-21, Auckland, New Zealand, February, 2006 
Masuyama, Y. \& Fukasawa T. (1997a). Full Scale Measurement of Sail Force and the Validation of Numerical Calculation Method, Proceedings of SNAME 13th Chesapeake Sailing Yacht Symposium, pp. 23-36, Annapolis, Maryland, USA, January, 1997

Masuyama, Y., Fukasawa T. \& Kitasaki T. (1997b). Investigations on Sail Forces by Full Scale Measurement and Numerical Calculation (Part 1: Steady Sailing Performance), Journal of Society of Naval Architects of Japan, Vol. 181, (June 1997), pp.1-13, ISSN 0514-8499, (in Japanese)

Masuyama, Y., Tahara, Y., Fukasawa, T. \& Maeda, N. (2007). Database of Sail Shapes vs. Sail Performance and Validation of Numerical Calculation for Upwind Condition, Proceedings of SNAME 18th Chesapeake Sailing Yacht Symposium, pp.11-31, Annapolis, Maryland, USA, March, 2007

Masuyama, Y., Tahara, Y., Fukasawa, T. \& Maeda, N. (2009). Database of Sail Shapes versus Sail Performance and Validation of Numerical Calculation for the Upwind Condition, Journal of Marine Science and Technology, JASNAOE, Vol. 14, No. 2, (2009), pp.137-160, ISSN 0948-4280

Milgram, J. H., Peters, D. B. \& Eckhouse, D.N., N. (1993). Modeling IACC Sail Forces by Combining Measurements with CFD, Proceedings of SNAME 11th Chesapeake Sailing Yacht Sympposium, pp.65-73, Annapolis, Maryland, USA, January, 1993

Pistolesi, E. (1937). Betrachtungen uber die gegenseitige Beeinflussung von Tragflügelsystemen. Gesammelte Vorträge der Hauptversammlung 1937 der LilienthalGesellschaft, pp.214-219

Tahara, Y. \& Stern, F. (1996). A Large-Domain Approach for Calculating Ship Boundary Layers and Wakes for Nonzero Froude Number, Journal of Computational Physics, Vol. 127, (1996), pp. 398-411

Tahara, Y. (1995). An Application of Two-Layer k- $\varepsilon$ Model to Ship Flow Computation, Journal of Society of Naval Architects of Japan, Vol. 177, (1995), pp. 161-176

Tahara, Y. (2008). A Reynolds-Averaged Navier-Stokes Equation Solver for Prediction of Ship Viscous Flow with Free Surface Effects, Proceedings of NAPA User Meeting 2008, Helsinki, Finland, May, 2008.

Tahara, Y., Tohyama, S. \& Katsui, T. (2006a). CFD-Based Multi-Objective Optimization Method for Ship Design, International Journal of Numerical Methods in Fluids, Vol. 52, (2006), pp. 449-527

Tahara, Y., Wilson, R., Carrica, P. \& Stern, F. (2006b). RANS Simulation of a Container Ship Using a Single-Phase Level Set Method with Overset Grids and Prognosis for Extension to Self-Propulsion Simulator, Journal of Marine Science and Technology, Vol. 11, No. 4, (2006), pp. 209-228

Thompson, J.F., Warsi, Z.U.A., \& Mastin, C.W. (1985). Numerical Grid Generation, Elsevier Science Publishing Co., Inc., NY. 


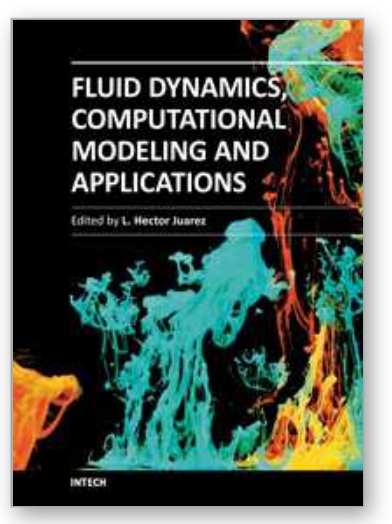

\author{
Fluid Dynamics, Computational Modeling and Applications \\ Edited by Dr. L. Hector Juarez
}

ISBN 978-953-51-0052-2

Hard cover, 660 pages

Publisher InTech

Published online 24, February, 2012

Published in print edition February, 2012

The content of this book covers several up-to-date topics in fluid dynamics, computational modeling and its applications, and it is intended to serve as a general reference for scientists, engineers, and graduate students. The book is comprised of 30 chapters divided into 5 parts, which include: winds, building and risk prevention; multiphase flow, structures and gases; heat transfer, combustion and energy; medical and biomechanical applications; and other important themes. This book also provides a comprehensive overview of computational fluid dynamics and applications, without excluding experimental and theoretical aspects.

\title{
How to reference
}

In order to correctly reference this scholarly work, feel free to copy and paste the following:

Y. Tahara, Y. Masuyama, T. Fukasawa and M. Katori (2012). Sail Performance Analysis of Sailing Yachts by Numerical Calculations and Experiments, Fluid Dynamics, Computational Modeling and Applications, Dr. L. Hector Juarez (Ed.), ISBN: 978-953-51-0052-2, InTech, Available from:

http://www.intechopen.com/books/fluid-dynamics-computational-modeling-and-applications/sail-performanceanalysis-of-sailing-yachts-by-numerical-calculations-and-experiments

\section{INTECH}

open science | open minds

\section{InTech Europe}

University Campus STeP Ri

Slavka Krautzeka 83/A

51000 Rijeka, Croatia

Phone: +385 (51) 770447

Fax: +385 (51) 686166

www.intechopen.com

\section{InTech China}

Unit 405, Office Block, Hotel Equatorial Shanghai

No.65, Yan An Road (West), Shanghai, 200040, China

中国上海市延安西路65号上海国际贵都大饭店办公楼405单元

Phone: +86-21-62489820

Fax: +86-21-62489821 
(C) 2012 The Author(s). Licensee IntechOpen. This is an open access article distributed under the terms of the Creative Commons Attribution 3.0 License, which permits unrestricted use, distribution, and reproduction in any medium, provided the original work is properly cited. 